\title{
AN INTRODUCTION TO KNOT FLOER HOMOLOGY AND CURVED BORDERED ALGEBRAS
}

\author{
ANTONIO ALFIERI AND JACKSON VAN DYKE
}

\begin{abstract}
We survey Ozsváth-Szabó's bordered approach to knot Floer homology. After a quick introduction to knot Floer homology, we introduce the relevant algebraic concepts ( $\mathcal{A}_{\infty}$-modules, type $D$-structures, box tensor product, etc.), we discuss partial Kauffman states, the construction of the boundary algebra, and sketch Ozsváth and Szabó's analytic construction of the type $D$-structure associated to an upper diagram. Finally we give an explicit description of the structure maps of the $D A$-bimodules of some elementary partial diagrams. These can be used to perform explicit computations of the knot Floer differential of any knot in $S^{3}$. The boundary DGAs $\mathcal{B}(n, k)$ and $\mathcal{A}(n, k)$ of $[6]$ are replaced here by an associative algebra $\mathcal{C}(n)$. These are the notes of two lecture series delivered by Peter Ozsváth and Zoltán Szabó at Princeton University during the summer of 2018.
\end{abstract}

\section{Contents}

1. Introduction 2

2. Knot Floer homology 3

2.1. Knot projections and Heegaard diagrams 3

2.2. The Floer setup of a Heegaard diagram 3

2.3. Intersection points and Kauffman states 4

2.4. The knot Floer chain complex 5

3. $\mathcal{A}_{\infty}$-modules and type $D$-structures 6

3.1. $\mathcal{A}_{\infty}$-algebras 6

3.2. $\mathcal{A}_{\infty}$-modules $\quad 7$

3.3. Type $D$-structures 8

3.4. Box tensor product $\quad 10$

3.5. Flat type $D$-structures as chain complexes 10

3.6. Curved $D A$-bimodules 11

3.7. Box tensor product and $D A$-bimodules 11

4. Partial Kauffman states $\quad 12$

4.1. Upper Kauffman states 12

4.2. Partial Kauffman states 13

4.3. Lower Kauffman states and gluing 13

5. Boundary algebras, curvature and matchings 14

5.1. The boundary algebra $\mathcal{C}(n) \quad 14$

5.2. Action of idempotents on partial Kauffman states 15

5.3. Curvature and matchings 16

6. Structure maps and the Gluing Theorem 16

6.1. The type $D$-structure of an upper diagram 16

$\begin{array}{ll}\text { 6.2. The Gluing Theorem } & 19\end{array}$

7. The bimodules of some elementary configurations 20 
7.2. No critical points, no gain 20

$\begin{array}{ll}\text { 7.3. Local Minima } & 20\end{array}$

$\begin{array}{ll}\text { 7.4. Crossings } & 21\end{array}$

7.5. A final remark 24

$\begin{array}{ll}\text { References } & 25\end{array}$

\section{INTRODUCTION}

A powerful paradigm in computer science is the so called Divide et Impera. It consists of dividing a complicated problem into smaller subproblems in order to solve them individually. In topology this translates to the cut and paste philosophy. More specifically: suppose that one wants to compute an invariant $D$ of some space $X$, then one can divide $X$ into pieces $P_{1}, \ldots, P_{N}$ and can compute some sort of related invariant $I$ for the pieces, and develop some gluing formula expressing $D(X)$ as a function of $I\left(P_{1}\right), \ldots, I\left(P_{N}\right)$. A typical example is when $D(X)$ is the fundamental group: in this case the invariants $I\left(P_{i}\right)$ are some inclusion maps, and the gluing principle is the Seifert-van Kampen Theorem.

In four-manifold topology, cut and paste techniques to compute Seiberg-Witten invariants were pioneered in [2] and eventually led to the definition of the Monopole Floer homology groups by Kronheimer and Mrowka [3]. In the case of the Heegaard Floer three-manifold groups an implementation of the cut and paste approach first appeared in [4]. In this work Lipshitz, Ozsváth and Thurston associate to three manifolds with boundary $Y_{1}$ and $Y_{2}$ a type $D$-structure $C F D\left(Y_{1}\right)$ and a $\mathcal{A}_{\infty}$-module $C F A\left(Y_{2}\right)$ respectively, so that $C F\left(Y_{1} \cup_{\partial}-Y_{2}\right)=C F D\left(Y_{1}\right) \otimes C F A\left(Y_{2}\right)$ where, whatever these algebraic structures are, $\square$ is some sort of tensor product operation that takes as input a type $D$-structure and an $\mathcal{A}_{\infty}$-module, and gives back a chain complex.

Ozsváth and Szabó developed a similar construction in the setting of knot Floer homology [6]. Given a knot $K \subset \mathbb{R}^{3}$ one can split it into two parts by means of a two-plane $z=t$. Denote by $K_{[t,+\infty)}=K \cap\{z \geq t\}$ the portion of $K$ lying above the plane $z=t$. Similarly denote by $K_{(-\infty, t]}=K \cap\{z \leq t\}$ the part lying below it. In [6] Ozsváth and Szabó associate to $K_{[t,+\infty)}$ and $\bar{K}_{(-\infty, t]}$ a type $D$-structure $\operatorname{DFK}\left(K_{[t,+\infty)}\right)$ and an $\mathcal{A}_{\infty}$-module $A F K\left(K_{(-\infty, t])}\right)$ so that

$$
C F K(K)=A F K\left(K_{(-\infty, t])}\right) \otimes D F K\left(K_{[t,+\infty)}\right) \text {. }
$$

More generally, they develop some sort of Morse theoretic approach one can apply to compute knot Floer homology: given $t_{1}<t_{2}$ they associate to $K_{\left[t_{1}, t_{2}\right]}=K \cap\left\{t_{1} \leq\right.$ $\left.z \leq t_{2}\right\}$ a type $D A$-structure ${ }^{1} D A F K\left(K_{\left[t_{1}, t_{2}\right]}\right)$ such that

$$
D F K\left(K_{\left[t_{1},+\infty\right)}\right)=\operatorname{DAFK}\left(K_{\left[t_{1}, t_{2}\right]}\right) \otimes D F K\left(K_{\left[t_{2},+\infty\right)}\right) .
$$

The scope of these lecture notes is to survey the algebraic language of $\mathcal{A}_{\infty}$-modules, type $D$ - and $D A$-structures, and sketch Ozsváth and Szabó's recent construction. We will only assume some familiarity with Floer homology and the Fukaya category as in [1] (for motivational reasons), and with Heegaard Floer homology as in [9].

\footnotetext{
${ }^{1} \mathrm{~A}$ type $D A$-structure is an algebraic structure that can be thought simultaneously as a type $D$-structure and an $\mathcal{A}_{\infty}$-module. Type $D$ - and $D A$-structures can be paired in order to produce a new type $D$-structure.
} 

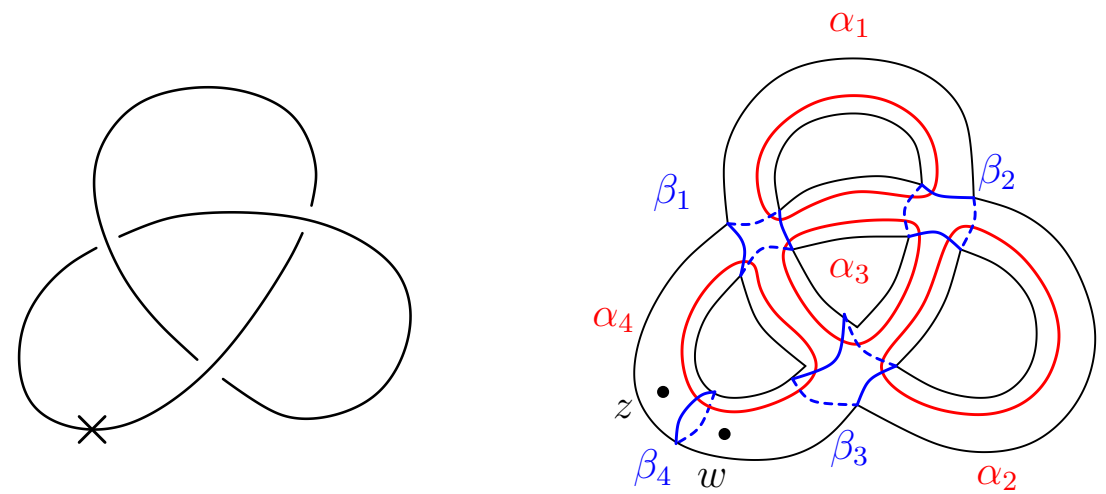

Figure 1. Doubly-pointed Heegaard diagram of the trefoil.

Acknowledgements. The authors would like to thank Peter Ozsváth, Zoltán Szabó and András Stipsicz, for many useful conversations. The first author is partially supported by the NKFIH Grant Élvonal (Frontier) KKP 126683 and from K112735.

\section{Knot Floer homology}

Knot Floer homology is a knot invariant introduced by Ozsváth and Szabó [7], and independently by Rasmussen [11]. We presently review their construction and some related variations.

2.1. Knot projections and Heegaard diagrams. A Heegaard diagram $(\Sigma, \boldsymbol{\alpha}, \boldsymbol{\beta})$ of $S^{3}$ together with two base points $w$ and $z \in \Sigma$ lying in the complement of the $\alpha$ - and the $\beta$-curves specifies an oriented knot $K \subset S^{3}$ as follows. Connect $w$ and $z$ in $\Sigma$ by an arc disjoint from the $\alpha$-curves, and push the resulting arc $a$ in the handlebody of the $\beta$-curves. Similarly, connect $w$ and $z$ by an arc disjoint from the $\beta$-curves, and push the resulting arc $b$ in the handlebody of the $\alpha$-curves. This produces a closed loop $K=a \cup b$ that can be oriented by taking $\partial a=z-w=-\partial b$.

One can also go in the opposite direction. Consider a knot $K \subset S^{3}$. Let $V$ be a planar projection of $K$. This is a connected, 4-valent planar graph. Thickening $V \subset \mathbb{R}^{2} \times 0 \subset \mathbb{R}^{3} \cup\{\infty\}$ we get a genus $g$ solid handlebody $U \subset S^{3}$ providing, together with its complement $U^{c}=\overline{S^{3}-V}$, a Heegaard splitting of $S^{3}$. As $\alpha$ curves of the corresponding Heegaard diagram (supported on $\Sigma=\partial U$ ) we choose the boundary of the compact complementary regions of $V$. (This provides a set of compressing circles for $\left.U_{\alpha}=U^{c}\right)$. As compressing circles of $U_{\beta}=U$ ( $\beta$-curves) we take one curve in correspondence with each crossing as suggested by Figure 1, and one further $\beta$-curve representing a meridian of $K$. By taking base points on the two sides of the $\beta$-curve corresponding to the meridian we get a doubly pointed Heegaard diagram $(\Sigma, \boldsymbol{\alpha}, \boldsymbol{\beta}, z, w)$ representing $K \subset S^{3}$.

Exercise 1. Prove that in the diagram associated to a knot projection there are as many $\alpha$-curves as $\beta$-curves, and that this number equals the genus.

2.2. The Floer setup of a Heegaard diagram. In [8] Ozsváth and Szabó associate to a Heegaard diagram $(\Sigma, \boldsymbol{\alpha}, \boldsymbol{\beta})$ a symplectic manifold $(M, \omega)$ together with two Lagrangians $L_{\boldsymbol{\alpha}}$ and $L_{\boldsymbol{\beta}} \subset M$. Their construction goes as follows. 
Endow $\Sigma$ with the structure of a Riemann surface and consider the space of degree $g$ divisors over $\Sigma$. This is the $g$-fold symmetric product $\operatorname{Sym}^{g}(\Sigma)$ of $\Sigma$

$$
\operatorname{Sym}^{g}(\Sigma)=\Sigma^{\times g} / \mathfrak{S}_{g}
$$

Here $\mathfrak{S}_{g}$ denotes the symmetric group on $g$ letters acting on the $g$-fold cartesian product $\Sigma^{\times g}$ by permutation of the coordinates. Although $\mathfrak{S}_{g}$ does not act freely, $\operatorname{Sym}^{g}(\Sigma)$ is a smooth complex manifold. Notice that the projection $\pi: \Sigma^{\times g} \rightarrow$ $\operatorname{Sym}^{g}(\Sigma)$ is an analytic covering with branching locus the fat diagonal

$$
\Delta=\left\{x_{1}+\cdots+x_{g} \in \operatorname{Sym}^{g}(\Sigma) \mid x_{i}=x_{j} \text { for some } i \neq j\right\} .
$$

Remark 1. At first glance it could be surprising that $\operatorname{Sym}^{g}(\Sigma)$ is a smooth manifold. This is clear if we look at the local picture. Consider the $g$-fold symmetric product of the complex plane $\operatorname{Sym}^{g}(\mathbb{C})$. This is the space of unordered $g$-tuples of complex numbers $\zeta=\left\{z_{1}, \ldots, z_{g}\right\}$ where each number can appear more than once. Sending an unordered $g$-tuple $\zeta=\left\{z_{1}, \ldots, z_{g}\right\}$ to the coefficients $\left(a_{0}, \ldots, a_{g-1}\right)$ of the (monic) polynomial $\prod_{i}\left(x-z_{i}\right)=x^{g}+a_{g-1} x^{g-1}+\cdots+a_{0}$ we get a map $\operatorname{Sym}^{g}(\mathbb{C}) \rightarrow \mathbb{C}^{g}$. It is easy to verify that, as a consequence of the Fundamental Theorem of Algebra, the map $\zeta \mapsto\left(a_{0}, \ldots, a_{g-1}\right)$ gives a homeomorphism $\operatorname{Sym}^{g}(\mathbb{C}) \simeq \mathbb{C}^{g}$.

One can turn $\operatorname{Sym}^{g}(\Sigma)$ into a symplectic manifold as follows. Pick an area form $\nu \in \Omega^{2}(T \Sigma)$ taming the complex structure of $\Sigma$. This induces a symplectic form $\nu^{\times g}=\nu \times \cdots \times \nu$ over $\Sigma^{\times g}$ taming the product complex structure of $\Sigma^{\times g}$. Now one would like to take the push forward $\omega=\pi_{*}\left(\nu^{\times g}\right)$ of $\nu^{\times g}$ through the covering projection $\pi: \Sigma^{\times g} \rightarrow \operatorname{Sym}^{g}(\Sigma)$. Unfortunately, this can't be done since the form $\omega$ would be somehow singular near to the branching locus of $\pi$ (the fat diagonal). This problem can be overcame using the work of Perutz [10].

Theorem 1. Suppose that $U$ is an open subset containing the fat diagonal $\Delta \subset$ $\operatorname{Sym}^{g}(\Sigma)$. Then there exists a Kähler form $\omega$ on $\operatorname{Sym}^{g}(\Sigma)$ that agrees on $\operatorname{Sym}^{g}(\Sigma) \backslash \bar{U}$ with the push-forward $\pi_{*}\left(\nu^{\times g}\right)$ of the product form. Furthermore, one can choose $\omega$ so that $[\omega]=\pi_{*}\left[\nu^{\times g}\right] \in H^{2}\left(\operatorname{Sym}^{g}(\Sigma) ; \mathbb{R}\right)$.

From the attaching circles we can form two smoothly embedded $g$-dimensional tori $\mathbb{T}_{\alpha}=\alpha_{1} \times \cdots \times \alpha_{g}$ and $\mathbb{T}_{\beta}=\beta_{1} \times \cdots \times \beta_{g}$ lying in the symmetric product Sym ${ }^{g}(\Sigma)$. Notice that $\mathbb{T}_{\alpha}$ are $\mathbb{T}_{\beta}$ are Lagrangian submanifolds of $\left(\operatorname{Sym}^{g}(\Sigma), \omega\right)$.

2.3. Intersection points and Kauffman states. The intersection points $\mathbb{T}_{\alpha} \cap \mathbb{T}_{\beta}$ of the $\alpha$ - and $\beta$-tori of a doubly pointed Heegaard diagram $(\Sigma, \boldsymbol{\alpha}, \boldsymbol{\beta}, z, w)$ associated to a knot projection can be interpreted combinatorially as follows.

Let $K$ be an oriented knot in $S^{3}$, and suppose that $V \subseteq \mathbb{R}^{2}$ is an oriented planar projection of $K$. A domain of $V$ is the closure of a connected component of its complement in $\mathbb{R}^{2}$. Let $C r(V)$ denote the set of crossings in the projection $V$ and $D_{0}(V)$ the set of its domains. Choose a distinguished arc of the projection (in figures such an arc will be marked with the symbol $\times$ ) and denote by $D(V)$ the set of those domains which are disjoint from the marked arc. A Kauffman state of the marked diagram $V$ is a bijection $\sigma: C r(V) \rightarrow D(V)$ such that $c \in \sigma(c)$ for all $c \in C r(V)$. Kauffman states are in obvious one to one correspondence with the intersection points of $\mathbb{T}_{\alpha} \cap \mathbb{T}_{\beta}$. A Kauffman state should be thought of as a choice of region for each crossing. In figures we will mark these regions with a black dot. See fig. 2 for an example. 


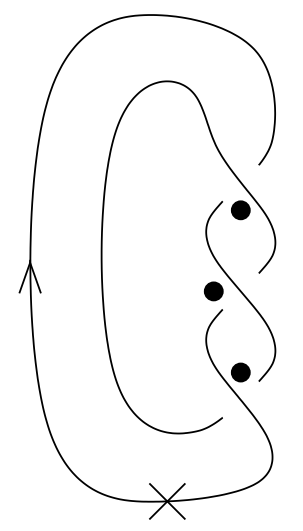

FiguRE 2. One of three Kauffman states for the left-handed trefoil knot.

2.4. The knot Floer chain complex. Given a genus $g$, doubly pointed Heegaard diagram $(\Sigma, \boldsymbol{\alpha}, \boldsymbol{\beta}, z, w)$, we can pick a symplectic form $\omega$ on $\operatorname{Sym}^{g}(\Sigma)$ as in Theorem 1, a generic path $J_{s} \in \mathcal{J}\left(\operatorname{Sym}^{g}(\Sigma)\right)$ of compatible almost-complex structures, and run the construction of Lagrangian Floer homology to get a differential on

$$
\widehat{C F}(\Sigma, \boldsymbol{\alpha}, \boldsymbol{\beta})=\bigoplus_{\mathbf{x} \in \mathbb{T}_{\alpha} \cap \mathbb{T}_{\beta}} \mathbb{Z}_{2} \cdot \mathbf{x} .
$$

In doing so one would immediately run into some technical problems [8]:

(1) if $g>2$ then $\pi_{2}\left(\operatorname{Sym}^{g}(\Sigma)\right)=\mathbb{Z}$. A generator $S \in \pi_{2}\left(\operatorname{Sym}^{g}(\Sigma), \mathbb{T}_{\alpha}\right)$ can be found as follows. Take a hyperelliptic involution $\tau$ on $\Sigma$ and fix a basepoint $z \in \Sigma$, then $y \in \Sigma / \tau=S^{2} \mapsto y+\tau(y)+(g-2) z \in \operatorname{Sym}^{g}(\Sigma)$ is a sphere representing $S$.

(2) Similarly, $\pi_{2}\left(\operatorname{Sym}^{g}(\Sigma), \mathbb{T}_{\alpha}\right)=\pi_{2}\left(\operatorname{Sym}^{g}(\Sigma), \mathbb{T}_{\beta}\right)=\mathbb{Z}$ for $g>2$.

In particular both bubbling and boundary degenerations can occur. To fix this, one can choose a basepoint $z \in \Sigma \backslash \boldsymbol{\alpha} \cup \boldsymbol{\beta}$ and count holomorphic disks only in $\operatorname{Sym}^{g}(\Sigma-z)=\operatorname{Sym}^{g}(\Sigma) \backslash V_{z}$, where $V_{z}=z \times \operatorname{Sym}^{g-1}(\Sigma)=\left\{\mathbf{x}=x_{1}+\cdots+x_{g} \in\right.$ $\operatorname{Sym}^{g}(\Sigma) \mid x_{i}=z$ for some $\left.i\right\}$. With this expedient we recover the conditions

$$
\pi_{2}\left(\operatorname{Sym}^{g}(\Sigma-z)\right)=0 \quad \pi_{2}\left(\operatorname{Sym}^{g}(\Sigma-z), \mathbb{T}_{\alpha}\right)=\pi_{2}\left(\operatorname{Sym}^{g}(\Sigma-z), \mathbb{T}_{\beta}\right)=0,
$$

and we can apply Floer's argument verbatim. We define the differential of $\widehat{C F}(\Sigma, \boldsymbol{\alpha}, \boldsymbol{\beta})$ to be

$$
\widehat{\partial} \mathbf{x}=\sum_{\mathbf{y} \in \mathbb{T}_{\alpha} \cap \mathbb{T}_{\beta}} \sum_{\substack{\phi \in \pi_{2}(\mathbf{x}, \mathbf{y}) \\ n_{z}(\phi)=0 \\ \mu(\phi)=1}} \#\left(\frac{\mathcal{M}(\phi)}{\mathbb{R}}\right) \cdot \mathbf{y},
$$

where $\pi_{2}(\mathbf{x}, \mathbf{y})$ denotes the set of homotopy classes of disks connecting $\mathbf{x}$ to $\mathbf{y}$ (i.e. maps $u: D^{2} \simeq[0,1] \times \mathbb{R} \rightarrow \operatorname{Sym}^{g}(\Sigma)$ such that $u(0 \times \mathbb{R}) \subseteq \mathbb{T}_{\boldsymbol{\alpha}}, u(1 \times \mathbb{R}) \subseteq \mathbb{T}_{\boldsymbol{\beta}}$, $\lim _{t \rightarrow-\infty} u(s+i t)=\mathbf{x}$ and $\left.\lim _{t \rightarrow+\infty} u(s+i t)=\mathbf{y}\right), \mu(\phi) \in \mathbb{Z}$ denotes the expected dimension of the moduli space $\mathcal{M}(\phi)$ of $J_{s}$-holomorphic representatives in such a class, and $n_{z}(\phi)=\#\left(\phi^{-1}\left(V_{z}\right)\right)$ the algebraic intersection of a homotopy class with the divisor $V_{z}=z \times \operatorname{Sym}^{g-1}(\Sigma)$.

Notice that $(\widehat{C F}(\Sigma, \boldsymbol{\alpha}, \boldsymbol{\beta}), \partial)$ is independent from the choice of the basepoint $w$. In fact its homology does not depend on the $\operatorname{knot}\left[8\right.$, Theorem 1.1]: $H_{*}(\widehat{C F}(\Sigma, \boldsymbol{\alpha}, \boldsymbol{\beta}), \partial)=$ 
$\mathbb{Z}_{2}$. The choice of the second basepoint $w$ determines a filtration over $\widehat{C F}(\Sigma, \boldsymbol{\alpha}, \boldsymbol{\beta})$. This is the Alexander filtration $A$ and it is characterized by the property

$$
A(\mathbf{x})-A(\mathbf{y})=n_{w}(\phi)-n_{z}(\phi)
$$

where $\mathbf{x}$ and $\mathbf{y} \in \mathbb{T}_{\alpha} \cap \mathbb{T}_{\beta}$ denote two generators, and $\phi \in \pi_{2}(\mathbf{x}, \mathbf{y})$. Looking at page $E_{1}$ of the associated spectral sequence we get a differential $\hat{\partial}_{K}$ over $\widehat{C F}(\Sigma, \boldsymbol{\alpha}, \boldsymbol{\beta})$. One explicitly computes

$$
\widehat{\partial}_{K} \mathbf{x}=\sum_{\mathbf{y} \in \mathbb{T}_{\alpha} \cap \mathbb{T}_{\beta}} \sum_{\begin{array}{c}
\phi \in \pi_{2}(\mathbf{x}, \mathbf{y}) \\
n_{w}(\phi)=0, n_{z}(\phi)=0 \\
\mu(\phi)=1
\end{array}} \#\left(\frac{\mathcal{M}(\phi)}{\mathbb{R}}\right) \cdot \mathbf{y},
$$

i.e. we only count holomorphic disks that do not cross both the divisor $V_{z}$ and $V_{w}$.

Another possibility is to count all $J_{s}$-holomorphic disks and record their intersection with both basepoints. Given a doubly pointed Heegaard diagram $(\Sigma, \boldsymbol{\alpha}, \boldsymbol{\beta}, z, w)$ of a knot $K \subset S^{3}$, we can form the chain complex freely generated by its Heegaard states over the ring $\mathbb{Z}_{2}[U, V] / U V$

$$
C F K(K):=\bigoplus_{\mathbf{x} \in \mathbb{T}_{\alpha} \cap \mathbb{T}_{\beta}} \mathbb{Z}_{2}[U, V] / U V \cdot \mathbf{x}
$$

This is equipped with the differential:

$$
\partial_{K}(\mathbf{x})=\sum_{\mathbf{y} \in \mathbb{T}_{\alpha} \cap \mathbb{T}_{\beta}} \sum_{\substack{\phi \in \pi_{2}(x, y) \\ \mu(\phi)=1}} \#\left(\frac{\mathcal{M}(\phi)}{\mathbb{R}}\right) U^{n_{z}(\phi)} V^{n_{w}(\phi)} \cdot \mathbf{y} .
$$

In [8] Ozsváth and Szabó proved that the chain homotopy type of $C F K(K)$ does not depend on the choice of the doubly pointed Heegaard diagram $(\Sigma, \boldsymbol{\alpha}, \boldsymbol{\beta}, z, w)$ of $K$, nor on the generic path of almost complex structures $J_{s} \in \mathcal{J}\left(\operatorname{Sym}^{g}(\Sigma)\right)$. We denote by $H F K(K)$ the homology of $C F K(K)$.

Imposing $U=0$ in the complex (instead of $U V=0$ ) we get a simplified version of the theory usually denoted by $H F K^{-}(K)$. Setting $U=V=0$ in $C F K(K)$ we get instead the homology of $\widehat{C F K}(K)=(\widehat{C F}(\Sigma, \boldsymbol{\alpha}, \boldsymbol{\beta}, z, w), \hat{\partial})$, usually denoted by $\widehat{H F K}(K)$.

\section{3. $\mathcal{A}_{\infty}$-Modules And type $D$-Structures}

3.1. $\mathcal{A}_{\infty}$-algebras. Let $R$ be a commutative ring with unit. We will often assume that $R$ has characteristic two, i.e. $1+1=0$. An $\mathcal{A}_{\infty}$-algebra $\mathcal{A}$ is a graded $R$ module together with (grading-preserving) linear maps $\mu_{j}: \mathcal{A}^{\otimes j} \rightarrow \mathcal{A}[2-j]$ defined for $j \geq 0$. These are required to satisfy the so called $\mathcal{A}_{\infty}$-relations:

$$
\sum_{i+j+\ell=n} \mu_{i+1+\ell} \circ\left(i d_{\mathcal{A}^{\otimes i}} \otimes \mu_{j} \otimes i d_{\mathcal{A}^{\otimes \ell}}\right)=0 .
$$

Here $\mathcal{A}[2-j]$ denotes the algebra $\mathcal{A}$ with degree shifted by $2-j$. That is, degree $d+2-j$ elements of $\mathcal{A}$ are declared to have grading $d$ in $\mathcal{A}[2-j]$. (This guarantees that $\mu_{1}: \mathcal{A} \rightarrow \mathcal{A}$ drops the grading by one for example.)

Exercise 2. Write down the $\mathcal{A}_{\infty}$-relations for $n=0,1,2$. Prove that in an $\mathcal{A}_{\infty^{-}}$ algebra $\partial \omega=0$ and $\partial^{2} a=\omega \cdot a+a \cdot \omega$, where we set $\omega=\mu_{0}(1), \partial x=\mu_{1}(x)$, and $x \cdot y=\mu_{2}(x \otimes y)$. 
$\mathcal{A}_{\infty}$-relations can be understood graphically as follows. Consider trees which have $n+1$ leaves, a preferred leaf (called the root), and exactly two vertices. Then each such tree $T$ represents a map $\mu_{T}: \mathcal{A}^{\otimes n} \rightarrow \mathcal{A}$ : the leaves of $T$ represent the inputs of $\mu_{T}$, the preferred leaf its output, and a vertex of valence $j$ represents the operation $\mu_{j}$. In this notation the $\mathcal{A}_{\infty}$-relations say that for all $n \geq 1$

$$
\sum_{T} \mu_{T}\left(a_{1} \otimes \cdots \otimes a_{n}\right)=\sum_{i=1}^{n} \mu_{n+1}\left(a_{1} \otimes \cdots \otimes a_{i-1} \otimes \omega \otimes a_{i} \otimes \cdots \otimes a_{n}\right),
$$

where we set $\omega=\mu_{0}(1)$ and the sum on the left is extended to all such trees with $n+1$ leaves. An example for $n=4$ is shown in Figure 3 .

Remark 2. $\mathcal{A}_{\infty}$-algebras generalize some classical algebraic structures. An $\mathcal{A}_{\infty^{-}}$ algebra with $\mu_{0}=0$ and $\mu_{j}=0$ for $j \geq 2$ is a chain complex, a differential graded algebra (DGA) is an $\mathcal{A}_{\infty}$-algebra with $\mu_{0}=0$ and $\mu_{j}=0$ for $j \geq 3$, while a graded associative algebra is simply an $\mathcal{A}_{\infty}$-algebra with $\mu_{j}=0$ for $j \neq 2$.

If $\mu_{0}: R \rightarrow \mathcal{A}$ is non-zero we say that $\mathcal{A}$ is a curved $\mathcal{A}_{\infty}$-algebra. In this case, $\mu_{0}(1)=\omega$ is called the curvature of $\mathcal{A}$. A curved $\mathcal{A}_{\infty}$-algebra with $\mu_{1}=0$ and $\mu_{j}=0$ for $j \geq 3$ is just a graded algebra $\mathcal{A}$ together with a preferred central element $\omega$, i.e. an element $\omega \in \mathcal{A}$ such that $\omega \cdot x=x \cdot \omega$. We will call these objects curved associative algebras.

Another way to understand the $\mathcal{A}_{\infty}$-relations is through the tensor algebra $T^{*}(\mathcal{A})=$ $\bigoplus_{i=0}^{\infty} \mathcal{A}^{\otimes i}$. Notice that the maps $\mu_{j}(j \geq 1)$ sum up to a map $\mu:{ }^{\infty} \mu_{j}: T^{*}(\mathcal{A}) \rightarrow \mathcal{A}$. Define $\bar{D}: T^{*}(\mathcal{A}) \rightarrow T^{*}(\mathcal{A})$ to be

$$
\bar{D}\left(a_{1} \otimes \cdots \otimes a_{n}\right)=\sum_{j=1}^{n} \sum_{\ell=1}^{n-j+1} a_{1} \otimes \cdots \otimes \mu_{j}\left(a_{\ell} \otimes \cdots \otimes a_{\ell+j-1}\right) \otimes \cdots \otimes a_{n} .
$$

Then the $\mathcal{A}_{\infty}$-relations can be written as $\mu \circ\left(\bar{D}+i_{\omega}\right)=0$ where $\omega$ represents the curvature of $\mathcal{A}$, and

$$
i_{\omega}\left(a_{1} \otimes \cdots \otimes a_{n}\right)=\sum_{i=0}^{n+1} a_{1} \otimes \cdots \otimes a_{i-1} \otimes \omega \otimes a_{i} \otimes \cdots \otimes a_{n} .
$$

In what follows we will agree that $\bar{D}(1)=\mu(1)=0$ and $i_{\omega}(1)=\omega$, where $1 \in R$ denotes the unit of the ground ring $R \subset T^{*}(\mathcal{A})=R \oplus \mathcal{A} \oplus(\mathcal{A} \otimes \mathcal{A}) \oplus \ldots$

3.2. $\mathcal{A}_{\infty}$-modules. An $\mathcal{A}_{\infty}$-module over an $\mathcal{A}_{\infty}$-algebra $\mathcal{A}$ is a graded, finitely generated $R$-module $M$ together with linear maps $m_{j}: M \otimes \mathcal{A}^{\otimes j-1} \rightarrow M[2-i]$ defined for $j=1,2, \ldots$ such that

$$
0=\sum_{i+j=n+1} m_{i} \circ\left(m_{j} \otimes i d_{\mathcal{A}^{\otimes i}}\right)+\sum_{i+j+k=n} m_{i+1+k} \circ\left(i d_{\mathcal{A}^{\otimes i}} \otimes \mu_{j} \otimes i d_{\mathcal{A}^{\otimes k}}\right)
$$

for all $n \geq 1$. These are the $\mathcal{A}_{\infty}$-relations for modules. As in the case of algebras, $\mathcal{A}_{\infty}$-relations of modules can be interpreted graphically. Consider trees which have $n+1$ inputs and a single output, where the furthest left input is privileged. Then each such tree $T$ represents a map $m_{T}: M \otimes \mathcal{A}^{\otimes n} \rightarrow M$. Again $\mathcal{A}_{\infty}$-relations say that for all $n \geq 0$

$$
\sum_{T} m_{T}\left(x \otimes a_{1} \otimes \cdots \otimes a_{n}\right)=\sum_{i=1}^{n} m_{n+1}\left(x \otimes a_{1} \otimes \cdots \otimes a_{i-1} \otimes \omega \otimes a_{i} \otimes \cdots \otimes a_{n}\right)
$$



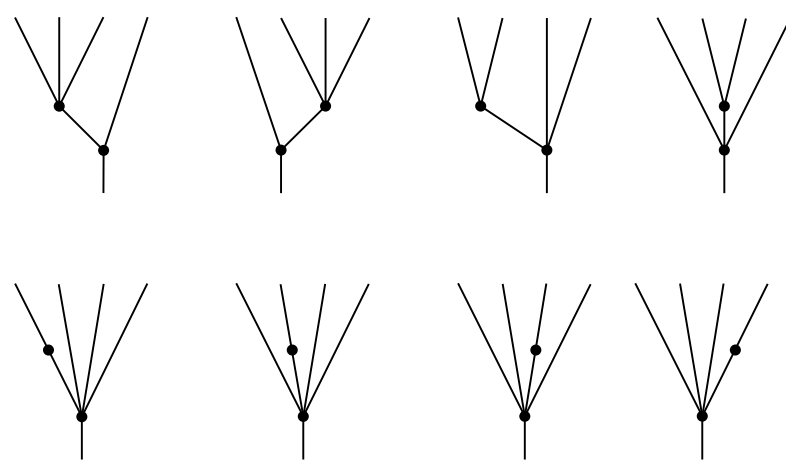

FiguRE 3. Operational trees for $n=4$. The rightmost tree on the first line represents $\mu_{3}\left(a_{1} \otimes a_{2} \otimes \mu_{2}\left(a_{3} \otimes a_{4}\right)\right)$. Notice that by collapsing the edge in between the two nodes of any tree on the left we get the four-legged tree on the right. In other words all operational trees are obtained from the tree on the right by blowing-up the central node in all possible fashions.

where the sum is extended over all above mentioned trees.

Alternatively, one can interpret the $\mathcal{A}_{\infty}$-relations by means of the tensor algebra $T^{*}(\mathcal{A})$ as follows. Define $m: T^{*}(\mathcal{A}) \otimes M \rightarrow M$ by summing up the $m_{j}$ maps, and consider the canonical co-multiplication $\Delta: T^{*}(\mathcal{A}) \rightarrow T^{*}(\mathcal{A}) \otimes T^{*}(\mathcal{A})$

$$
\Delta\left(a_{1} \otimes \cdots \otimes a_{n}\right)=\sum_{i+j=n}\left(a_{1} \otimes \ldots a_{i}\right) \otimes\left(a_{i+1} \otimes \cdots \otimes a_{n}\right) .
$$

Then the $\mathcal{A}_{\infty}$-relations can be graphically interpreted as insisting that

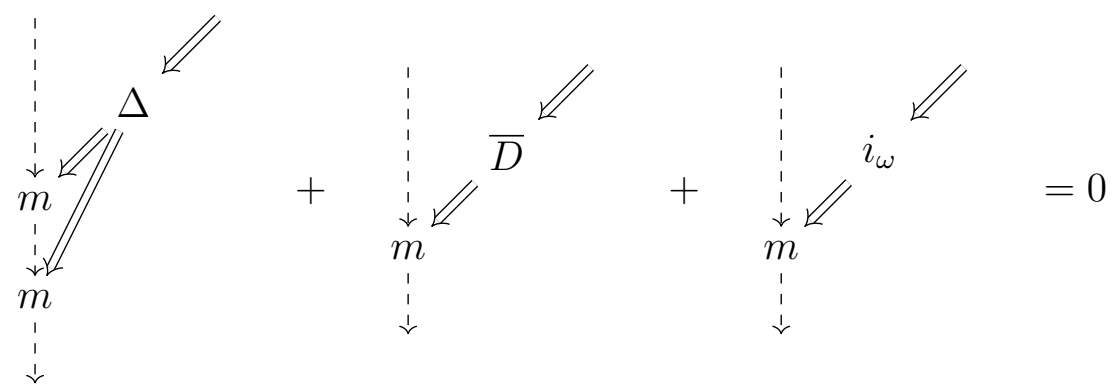

where dashed arrows represent module elements, and double arrows indicate elements of the tensor algebra $T^{*}(\mathcal{A})$. Recall $\bar{D}$ and $i_{\omega}$ are defined in $(1)$ and $(2)$.

3.3. Type $D$-structures. Let $\mathcal{A}$ be an $\mathcal{A}_{\infty}$-algebra with curvature $\omega$, and $X$ a graded, finitely generated $R$-module. Given a linear map $\delta: X \rightarrow \mathcal{A}[1] \otimes X$ we can form maps $\delta^{j}: X \rightarrow \mathcal{A}[1]^{\otimes j} \otimes X$ for $j=1,2, \ldots$ by simply iterating $\delta$ on the rightmost argument. We say that $(X, \delta)$ forms a ( $\omega$-curved) type $D$-structure provided that the structure equation is satisfied:

$$
\sum_{j=1}^{\infty}\left(\mu_{j} \otimes \operatorname{id}_{X}\right) \circ \delta^{j}=\omega \otimes \operatorname{id}_{X} .
$$

In terms of the structure map $\mu=T^{*}(\mathcal{A}) \rightarrow \mathcal{A}$ of the $\mathcal{A}_{\infty}$-algebra $\mathcal{A}$ this is the same as requiring $\left(\mu \otimes i d_{X}\right) \circ \bar{\delta}=\omega \otimes i d_{X}$, where $\bar{\delta}: X \rightarrow T^{*}(\mathcal{A}) \otimes X$ is defined 
by $\bar{\delta}(x)=\sum_{j=0}^{\infty} \delta^{j}(x)$. In what follows we will be mainly interested in $D$-structures over curved associative algebras. In this case the structure equation simplifies to

$$
\left(\mu_{2} \otimes \operatorname{id}_{X}\right) \circ\left(\operatorname{id}_{\mathcal{A}} \otimes \delta\right) \circ \delta=\omega \otimes \operatorname{id}_{X} .
$$

Lemma 1. Let $(X, \delta)$ be a type D-structure, then
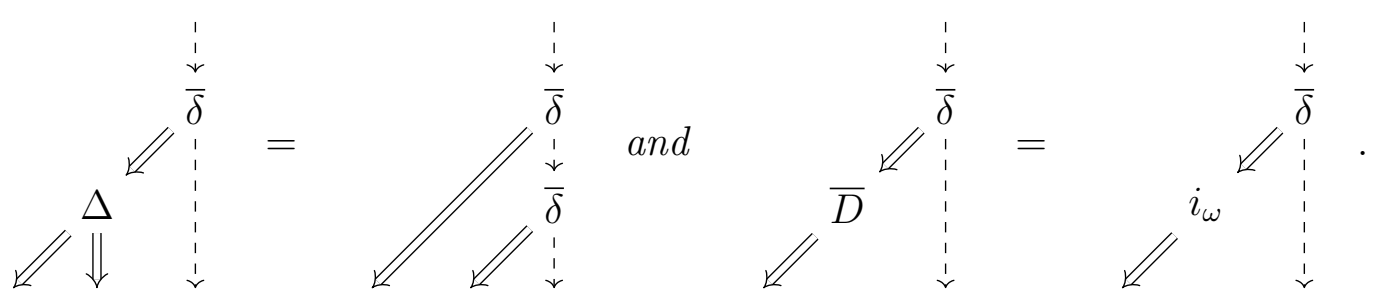

Proof. The first identity is a consequence of the fact that $\delta^{i+j}=\left(i d_{\mathcal{A}^{\otimes j}} \otimes \delta^{i}\right) \circ \delta^{j}$. The second identity is in fact equivalent to the structure equation. We give a proof in the case of curved associative algebras just to provide an example of direct computation.

Let $\mathbf{x}_{1}, \ldots, \mathbf{x}_{m}$ be a basis of $X$. Write:

$$
\delta\left(\mathbf{x}_{i}\right)=\sum_{j=1}^{m} \xi_{i j} \otimes \mathbf{x}_{j}
$$

for some constants $\xi_{i j} \in \mathcal{A}$. Notice that in linear coordinates the structure equation $\left(\mu_{2} \otimes \operatorname{id}_{X}\right) \circ\left(\operatorname{id}_{\mathcal{A}} \otimes \delta\right) \circ \delta=\omega \otimes \mathrm{id}_{X}$ translates to

$$
\sum_{\ell=1}^{m} \xi_{i \ell} \cdot \xi_{\ell j}= \begin{cases}\omega & \text { if } i=j \\ 0 & \text { otherwise }\end{cases}
$$

Using the coefficients $\xi_{i j}$ we obtain the following expression for $\bar{\delta}\left(\mathbf{x}_{i}\right)$

$$
\bar{\delta}\left(\mathbf{x}_{i}\right)=1 \otimes \mathbf{x}_{i}+\sum_{j} \xi_{i j} \otimes \mathbf{x}_{j}+\sum_{j, \ell}^{m} \xi_{i j} \otimes \xi_{j \ell} \otimes \mathbf{x}_{\ell}+\sum_{j, \ell, k}^{m} \xi_{i j} \otimes \xi_{j \ell} \otimes \xi_{\ell k} \otimes \mathbf{x}_{k}+\ldots
$$

Thus, applying $\bar{D}$ gives us

$$
\begin{aligned}
\bar{D} \otimes \operatorname{id}_{X}\left(\bar{\delta}\left(\mathbf{x}_{i}\right)\right) & =\sum_{j, \ell} \xi_{i j} \cdot \xi_{j \ell} \otimes \mathbf{x}_{\ell} \\
& +\sum_{j, \ell, k} \xi_{i j} \cdot \xi_{j \ell} \otimes \xi_{\ell k} \otimes \mathbf{x}_{k}+\sum_{j, \ell, k} \xi_{i j} \otimes \xi_{j \ell} \cdot \xi_{\ell k} \otimes \mathbf{x}_{k}+\ldots \\
& =\sum_{\ell}\left(\sum_{j} \xi_{i j} \cdot \xi_{j \ell}\right) \otimes \mathbf{x}_{\ell} \\
& +\sum_{\ell, k}\left(\sum_{j} \xi_{i j} \cdot \xi_{j \ell}\right) \otimes \xi_{\ell k} \otimes \mathbf{x}_{k}+\sum_{j, k} \xi_{i j} \otimes\left(\sum_{\ell} \xi_{j \ell} \cdot \xi_{\ell k}\right) \otimes \mathbf{x}_{k}+\cdots
\end{aligned}
$$

Imposing the curvature equation (5), we conclude that

$$
\begin{aligned}
\bar{D} \otimes \operatorname{id}_{X}\left(\bar{\delta}\left(\mathbf{x}_{i}\right)\right) & =\omega \otimes \mathbf{x}_{i}+\sum_{k} \omega \otimes \xi_{i k} \otimes \mathbf{x}_{k}+\sum_{k} \xi_{i j} \otimes \omega \otimes \mathbf{x}_{k}+\cdots \\
& =i_{\omega} \otimes i d_{X}\left(1 \otimes \mathbf{x}_{i}\right)+i_{\omega} \otimes i d_{X}\left(\delta\left(\mathbf{x}_{i}\right)\right)+\cdots
\end{aligned}
$$

and we are done. 
3.4. Box tensor product. There is a natural pairing between $\mathcal{A}_{\infty}$-modules and type $D$-structures. Suppose that $X$ is a type $D$-structure, and $M$ an $\mathcal{A}_{\infty}$-module over a curved associative algebra $\mathcal{A}$. Then we can equip the tensor product $M \otimes_{R} X$ with the endomorphism

$$
D=\sum_{j=0}^{\infty}\left(m_{j+1} \otimes \mathrm{id}_{X}\right) \circ\left(\operatorname{id}_{M} \otimes \delta^{j}\right) .
$$

Notice that this might not be well-defined since the sum can be infinite. This is not the case if, for example, either $M$ or $X$ is operationally bounded, i.e. $m_{j} \equiv 0$ or $\delta^{j} \equiv 0$ for large values of the indices.

Theorem 2. $\left(M \otimes_{R} X, D\right)$ is a chain complex, i.e. $D^{2}=0$.

Proof. By the definition of $\bar{\delta}$ and $\Delta$, we can write:

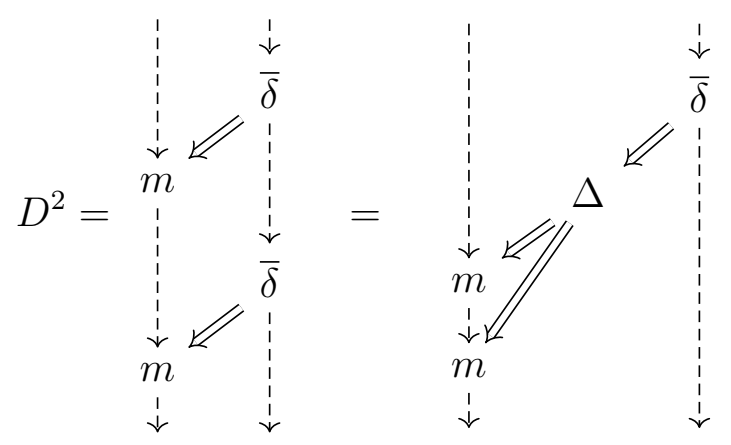

Now according to the structure equation (3) for the $\mathcal{A}_{\infty}$ module $M$ we can write

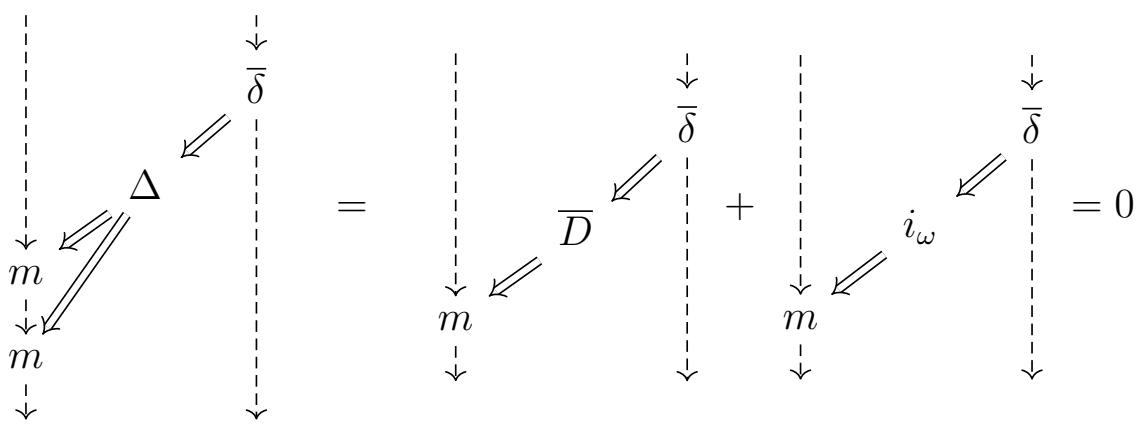

where this last expression is 0 by Lemma 1 .

We define $N \otimes X=\left(N \otimes_{R} X, \delta\right)$ to be the box tensor product of $N$ and $X$.

3.5. Flat type $D$-structures as chain complexes. A type $D$-structure $(X, \delta)$ over a curved associative algebra $(\mathcal{A}, \omega)$ is called flat if $\omega=0$. A flat type $D$ structure can be seen as a chain complex: extend the coefficients of $X$ by taking $X_{\mathcal{A}}=\mathcal{A} \otimes X$ and set $\partial=\left(\mu_{2} \otimes i d_{X}\right) \circ\left(i d_{\mathcal{A}} \otimes \delta\right)$. If $X$ is free with basis $B$ then:

$$
X_{\mathcal{A}}=\bigoplus_{\mathbf{x} \in B} \mathcal{A} \cdot \mathbf{x} \quad \text { and } \quad \partial(\mathbf{x})=\sum_{\mathbf{y} \in B} \xi_{\mathbf{x}, \mathbf{y}} \cdot \mathbf{y},
$$

where $\xi_{\mathbf{x}, \mathbf{y}} \in \mathcal{A}$ denote the structure constants of $\delta$, i.e.

$$
\delta(\mathbf{x})=\sum_{\mathbf{y} \in B} \xi_{\mathbf{x}, \mathbf{y}} \otimes \mathbf{y} .
$$


In what follows we will tacitly consider flat type $D$-structures as chain complexes in the sense specified above.

Remark 3. In general, if $\omega \neq 0$ one has $\partial^{2}(x)=\omega \cdot x$. To fix this defect and get $\partial^{2}=0$ one can take $X_{\mathcal{A}}=X \otimes \mathcal{A} / I \cdot X \otimes \mathcal{A}$ as a module over $\mathcal{A}_{I}=\mathcal{A} / I \mathcal{A}$, where $I \subset \mathcal{A}$ denotes the (principal) ideal generated by $\omega$.

3.6. Curved $D A$-bimodules. Let $\left(\mathcal{A}, \omega_{\text {new }}\right)$ and $\left(\mathcal{B}, \omega_{\text {old }}\right)$ be two curved associative algebras possibly over two different rings $R_{1}$ and $R_{2}$. A type $D A$-bimodule $N$ is a graded bimodule over $R_{1}$ and $R_{2}$ (acting respectively on the left and the right) together with linear maps

$$
\delta_{1+j}: N \otimes_{R_{2}} \mathcal{B}^{\otimes j} \rightarrow \mathcal{A} \otimes_{R_{1}} N
$$

defined for $j \geq 0$. These are required to satisfy the structure equations:
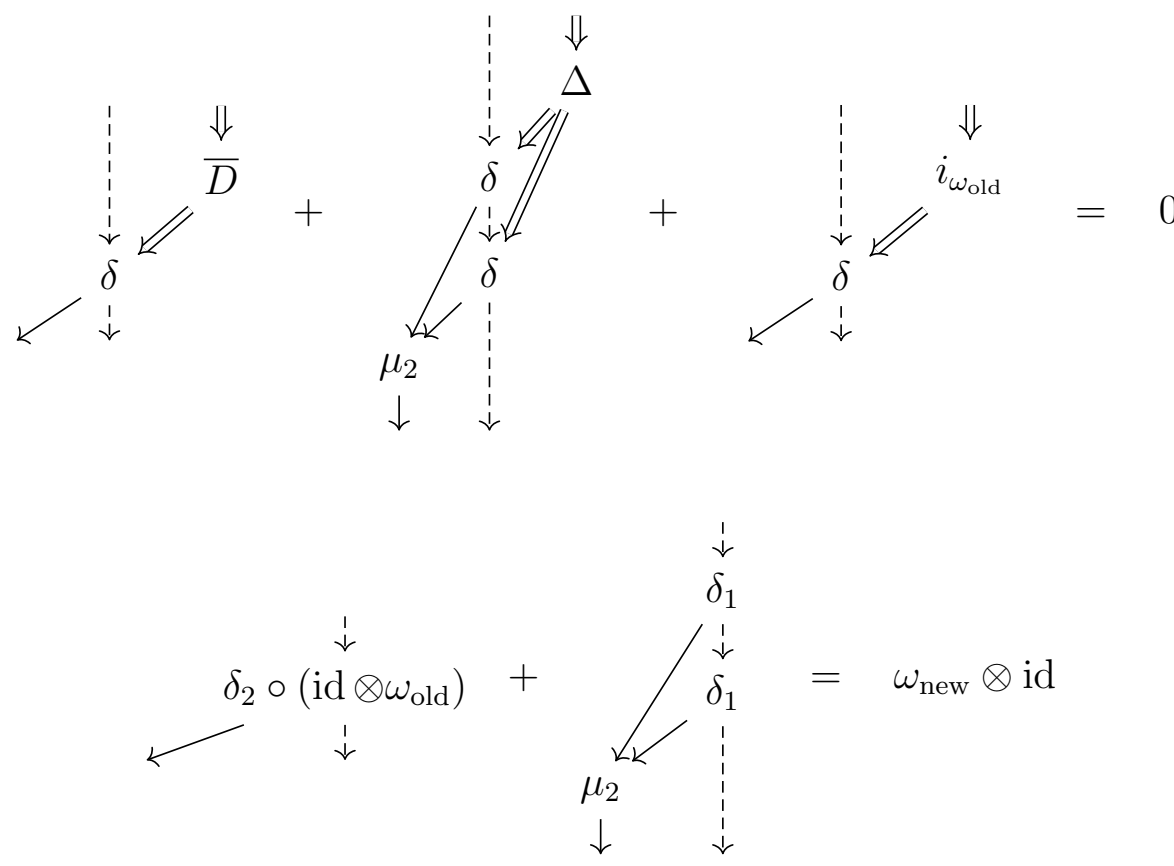

where $\delta=\sum_{j=1}^{\infty} \delta_{j}$. Here double arrows denote elements of the tensor algebra $T^{*}(\mathcal{B})$, dashed arrows elements of $N$, and plain arrows elements of $\mathcal{A}$.

3.7. Box tensor product and $D A$-bimodules. Just as in the case of type $D$ structures and $\mathcal{A}_{\infty}$-modules, one can pair type $D$-structures with $D A$-bimodules, and $D A$-bimodules with one another.

Let $\mathcal{A}$ and $\mathcal{B}$ be $\mathcal{A}_{\infty}$-algebras, $\left(X, \delta_{X}\right)$ a $D$-structure over $\mathcal{B}$, and $\left(N, \delta_{1}, \delta_{2}, \ldots\right)$ a $D A$-bimodule over $\mathcal{A}$ and $\mathcal{B}$. On the tensor product $N \otimes X$ we define

$$
\delta=\sum_{j=0}^{+\infty}\left(\delta_{1+j} \otimes i d_{X}\right) \circ\left(i d_{N} \otimes \delta_{X}^{j}\right) .
$$

This gives a map $\delta: N \otimes X \rightarrow \mathcal{A} \otimes N \otimes X$. As in Theorem 3 one can check that $\delta$ is the structure map of a type $D$-structure on $N \otimes X$. We define the type $D$-structure $N \otimes X=(N \otimes X, \delta)$ to be the box tensor product of $N$ and $X$.

Similarly, we can pair $D A$-bimodules with one another. Let $\left(M, \delta_{1}^{M}, \delta_{2}^{M}, \ldots\right)$ be a $D A$-bimodule over $\mathcal{A}$ and $\mathcal{C}$, and $\left(N, \delta_{1}^{N}, \delta_{2}^{N}, \ldots\right)$ a $D A$-bimodule over $\mathcal{C}$ and $\mathcal{B}$. Then 

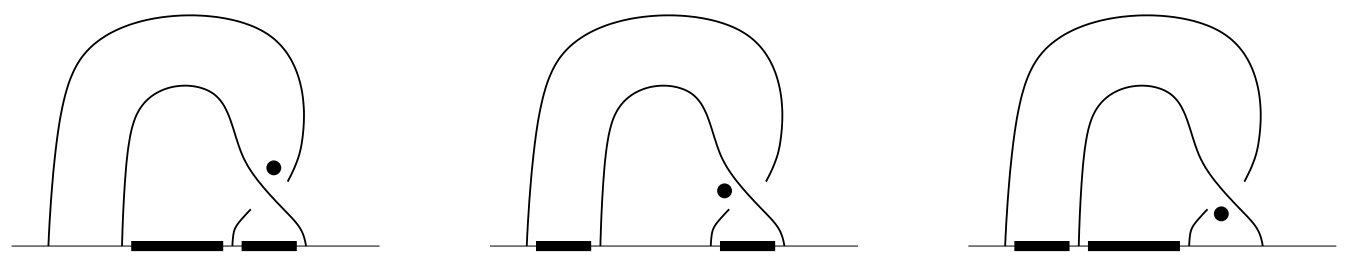

FiguRE 4. The three upper Kauffman states for this upper diagram. Note that this is the upper diagram from the projection of the lefthanded trefoil of Figure 2.

we can endow $M \otimes N$ with the $D A$-bimodule map $\delta_{1+*}^{M \otimes N}: N \otimes T^{*}(\mathcal{B}) \rightarrow \mathcal{A}[1] \otimes N$ defined graphically by

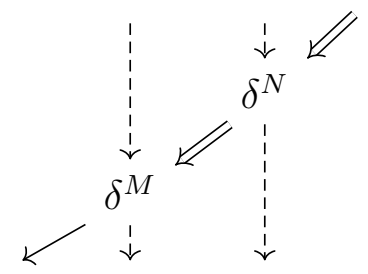

where $\delta^{M}=\sum_{j=1}^{\infty} \delta_{j}^{M}$ and $\delta^{N}=\sum_{j=1}^{\infty} \delta_{j}^{N}$. The resulting $D A$-bimodule (over $\mathcal{A}$ and $\mathcal{B}$ ) is the box tensor product of $M$ and $N$ and will also be denoted by $M \otimes N$.

\section{Partial Kauffman states}

Let $K \subset \mathbb{R}^{3}$ be a knot. Denote by $\pi(x, y, z)=(x, z)$ the projection on the $x z$-plane and by $h(x, y, z)=z$ the height function. Suppose that $\left.\pi\right|_{K}$ is a regular projection and that $\left.h\right|_{K}$ is a Morse function with all local maxima at $h=+10$ and global minimum at $h=-10$. Fix $t \in(-10,10)$ and consider the plane $z=t$. This intersects $K$ transversely in $2 n$ points and splits it in two parts: $K_{[t,+\infty)}=K \cap\{z \geq$ $t\}$ the part lying above the plane, and $K_{(-\infty, t]}=K \cap\{z \leq t\}$ the part lying below it. Recall our scope is to associate to $K_{[t,+\infty)}$ a type $D$-structure $D F K\left(K_{[t,+\infty)}\right)$ and to $K_{(-\infty, t]}$ an $\mathcal{A}_{\infty}$-module $A F K\left(K_{(-\infty, t]}\right)$ so that

$$
C F K(K)=A F K\left(K_{(-\infty, t]}\right) \otimes D F K\left(K_{[t,+\infty)}\right) .
$$

In fact, we associate to the portion of $K$ lying in between two parallel planes $K_{\left[t_{1}, t_{2}\right]}=$ $K \cap\left\{t_{1} \leq z \leq t_{2}\right\}$ a $D A$-bimodule $D A F K\left(K_{\left[t_{1}, t_{2}\right]}\right)$ so that

$$
\operatorname{DFK}\left(K_{\left[t_{1},+\infty\right)}\right)=\operatorname{DAFK}\left(K_{\left[t_{1}, t_{2}\right]}\right) \otimes D F K\left(K_{\left[t_{2},+\infty\right)}\right) .
$$

In this section we describe the generators of the vector spaces underlying $D F K$, $D A F K$ and $A F K$ in terms of combinatorial data associated to the projection.

4.1. Upper Kauffman states. Let us start with $D F K\left(K_{[t,+\infty)}\right)$. Consider the projection $D_{[t,+\infty)}=\pi(K) \cap \mathbb{R} \times[t,+\infty)$ of the portion of $K$ lying above the plane $z=t$ on the plane $y=0$. This is the upper diagram of the slice $z=t$. Choose $t \in(-10,10)$ so that there are no crossings or local minima at level $t$.

A local state, is a subset of $n$ elements of the set $\{1, \cdots, 2 n-1\}$. This corresponds to choosing $n$ of the $2 n-1$ bounded intervals separated by the strands on the line $z=t$. In figures we will denote the choice of such spaces with a black bar. See fig. 4 for an example. 
An upper Kauffman state is a pair $(\kappa, x)$, where $x$ is an local state, and $\kappa$ is a function associating to each of the crossings in the upper diagram a connected component of $\mathbb{R} \times[t,+\infty) \backslash D_{[t,+\infty)}$. For an upper Kauffman state we require the following conditions to be met:

(1) for each crossing $c$ of $D_{[t,+\infty)}$ the region $\kappa(c)$ is one of the (at most four) regions having $c$ as boundary corner,

(2) $\kappa$ is injective and does not allow the unbounded region to be occupied,

(3) the unbounded region meets none of the intervals in the local state $x$,

(4) for every bounded, unoccupied region, there exists exactly one interval in $x$ which bounds the region.

In figures the occupied regions will have a black dot. Define $D F K\left(K_{[t,+\infty)}\right)$ to be the vector space generated over $\mathbb{Z}_{2}$ by the upper Kauffman states.

4.2. Partial Kauffman states. Now instead of considering an upper diagram, consider a partial knot diagram. This consists of $D_{\left[t_{1}, t_{2}\right]}=\pi(K) \cap \mathbb{R} \times\left[t_{1}, t_{2}\right]$ the portion of the front projection of $K$ between two horizontal planes $z=t_{1}$ and $z=t_{2}$ for $t_{1}<t_{2}$. A partial Kauffman state is a triple $(\kappa, x, y)$ where $x$ is a local state of the $z=t_{1}$ slice, $y$ is a local state of the $z=t_{2}$ slice, and $\kappa$ is a map associating to each of the crossings of $D_{\left[t_{1}, t_{2}\right]}$ a bounded connected component of $\mathbb{R} \times\left[t_{1}, t_{2}\right] \backslash D_{\left[t_{1}, t_{2}\right]}$. For a partial Kauffman state the following conditions are required to hold:

(1) $\kappa$ is injective, and $c \in \partial \kappa(c)$ for each crossing $c$ of $D_{\left[t_{1}, t_{2}\right]}$,

(2) if a region $\mathcal{S}$ is occupied by $\kappa$, then $y$ contains all the intervals at $z=t_{2}$ intersecting $\mathcal{S}$, and $x$ contains none of the intervals at $z=t_{1}$ lying on $\mathcal{S}$,

(3) if $\mathcal{S}$ is unoccupied by $\kappa$, then either:

(a) $y$ contains all but one of the intervals at $z=t_{2}$ intersecting $\mathcal{S}$, and $x$ contains none of the intervals at $z=t_{1}$ intersecting $\mathcal{S}$

(b) or $y$ contains all of the intervals at $z=t_{2}$ intersecting $\mathcal{S}$, and $x$ contains exactly one of the intervals at $z=t_{1}$ intersecting $\mathcal{S}$.

We denote by $\operatorname{DAFK}\left(K_{\left[t_{1}, t_{2}\right]}\right)$ the vector space generated over $\mathbb{Z}_{2}$ by the partial Kauffman states. In figures the occupied regions will have a black dot.

4.3. Lower Kauffman states and gluing. There is an obvious notion of gluing between upper and partial Kauffman states: given a Kauffman state $\left(\kappa_{\mathcal{U}}, y\right)$ of $D_{\left[t_{2},+\infty\right)}$, and a Kauffman state $\left(\kappa_{\mathcal{P}}, x, y\right)$ of $D_{\left[t_{1}, t_{2}\right]}$ we can form a Kauffman state $\left(\kappa_{\mathcal{P}}, x, y\right) *\left(\kappa_{\mathcal{U}}, y\right)=\left(\kappa_{\mathcal{P}} * \kappa_{\mathcal{U}}, x\right)$ of $D_{\left[t_{1},+\infty\right)}$ by simply setting $\kappa_{\mathcal{U}} * \kappa_{\mathcal{P}}(c)=\kappa_{\mathcal{U}}(c)$ if $c$ is a crossing of $D_{\left[t_{1},+\infty\right)}$, and $\kappa_{\mathcal{U}} * \kappa_{\mathcal{P}}(c)=\kappa_{\mathcal{P}}(c)$ otherwise.

Exercise 3. Choose $\epsilon>0$ so that $D_{[-10+\epsilon, t]}=\pi(K) \cap \mathbb{R} \times[-10+\epsilon, t]$ does not contain the global minimum as in Figure 5. Suppose that we have an upper Kauffman state $\left(\kappa_{\mathcal{U}}, y\right)$ of $D_{[t,+\infty}$, and a partial Kauffman state $\left(\kappa_{\mathcal{L}}, x, y\right)$ of the middle region $D_{[-10+\epsilon, t]}$. Show that the gluing $\kappa_{\mathcal{L}} * \kappa_{\mathcal{U}}$ defines a Kauffman state of the knot at large. Show that all the Kauffman states of $K$ arise this way.

Fix $\epsilon>0$ sufficiently small so that $D_{[-10+\epsilon, t]}$ does not contain the global minimum, and define $A F K\left(D_{(-\infty, t]}\right)$ to be the $\mathbb{Z}_{2}$-vector space generated by the partial Kauffman states of $D_{[-10+\epsilon, t]}$, i.e. set $A F K\left(D_{(-\infty, t]}\right)=D A F K\left(D_{[-10+\epsilon, t]}\right)$. The generators of $A F K\left(D_{(-\infty, t]}\right)$ are called lower Kauffman states. 


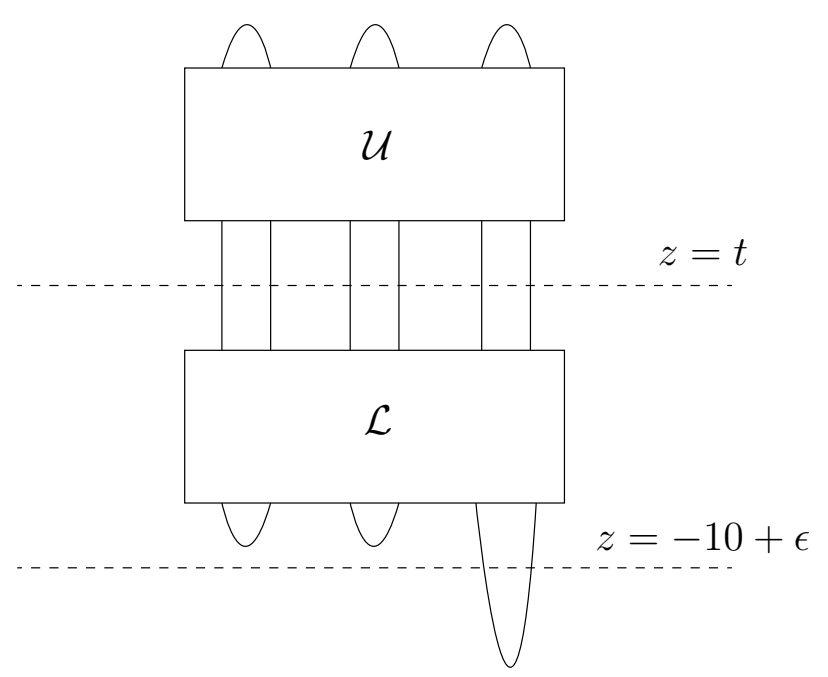

FiguRE 5. Knot divided into the portion $\mathcal{U}$ and $\mathcal{L}$.

\section{Boundary ALGEBras, CURVAture And MATCHings}

5.1. The boundary algebra $\mathcal{C}(n)$. The type $D$-structures and $\mathcal{A}_{\infty}$-modules associated to upper and lower diagrams will be defined over a certain associative algebra $\mathcal{C}(n)$ which only depends on the number of endpoints of the diagrams. The algebra $\mathcal{C}(n)$ is the quotient of a larger algebra $\mathcal{C}_{0}(n)$ which we will define presently.

As a vector space over $\mathbb{Z}_{2}, \mathcal{C}_{0}(n)$ is generated by the set of triples:

$$
\left(x, y, U_{1}^{a_{1}} \cdots U_{2 n}^{a_{2 n}}\right)
$$

where $x, y$ are two local states, and $U_{1}^{a_{1}} \cdots U_{2 n}^{a_{2 n}}$ is a monomial in $\mathbb{F}\left[U_{1}, \cdots, U_{2 n}\right]$.

Define the weight vector $w\left(x, y, U_{1}^{a_{1}} \cdots U_{2 n}^{a_{2 n}}\right) \in \mathbb{Z}^{2 n}$ of a generator by setting

$$
w\left(x, y, U_{1}^{a_{1}} \cdots U_{2 n}^{a_{2 n}}\right)=\frac{1}{2} v(x, y)+\left(a_{1}, \cdots, a_{2 n}\right)
$$

where the $i$ th component $v_{i}(x, y)$ of $v(x, y) \in \mathbb{Z}^{2 n}$ is given by:

$$
v_{i}(x, y)=|\#\{j \in x \mid i \leq j\}-\#\{k \in y \mid i \leq k\}| .
$$

This can be thought of as a vector which keeps track of how $x$ changes into $y$. See Figure 6 for an example. We define the product $a \cdot b$ of two generators $a=$ $\left(x, y, U_{1}^{a_{1}} \cdots U_{2 n}^{a_{2 n}}\right)$ and $b=\left(s, r, U_{1}^{b_{1}} \cdots U_{2 n}^{b_{2 n}}\right)$ of $\mathcal{C}_{0}(n)$ to be zero if $y \neq s$, and

$$
a \cdot b=\left(x, r, U_{1}^{t_{1}} \cdots U_{2 n}^{t_{2 n}}\right)
$$

if $y=s$, where $t_{1}, \cdots, t_{2 n}$ are chosen so that $w(a \cdot b)=w(a)+w(b)$.

For the local state $x$ set $I_{x}=(x, x, 1)$. A straightforward computation shows that

$$
I_{x} \cdot I_{y}=\left\{\begin{array}{ll}
I_{x} & x=y \\
0 & x \neq y
\end{array} .\right.
$$

In particular, $I_{x}$ is an idempotent element. In fact, $\left\{I_{x} \mid x\right.$ is a local state $\}$ generate the ring of idempotents of $\mathcal{C}_{0}(n)$. This ring will be denoted by $I(n)$. Notice that $\mathcal{C}_{0}(n)$ is a unital algebra over $\mathbb{F}\left[U_{1}, \cdots, U_{2 n}\right]$, where

$$
1=\sum_{x \in \text { local states }} I_{x}, \quad \text { and } \quad U_{i}=\sum_{x \in \text { local states }}\left(x, x, U_{i}\right) \text { for } i=1, \ldots, 2 n .
$$




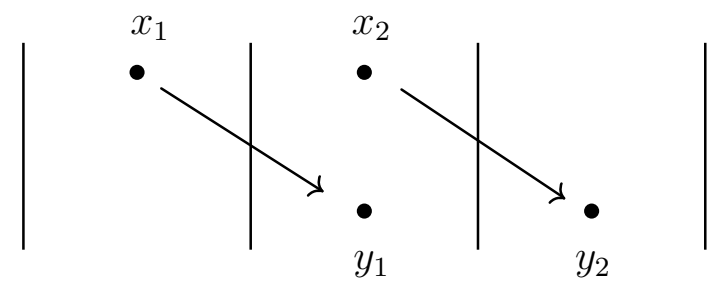

Figure 6 . Take $n=2$. In this case $v(x, y) \in \mathbb{Z}^{4}$. In particular, $v(x, y)=(|2-2|,|1-2|,|0-1|,|0-0|)=(0,1,1,0)$. This should be thought of as a vector which keeps track of how to 'get' from one local state to another by counting which boundaries are traversed.

The algebra $\mathcal{C}(n)$ is defined by imposing on $\mathcal{C}_{0}(n)$ the following relations:

(R-1) $\left(x, y, U_{1}^{t_{1}} \cdots U_{2 n}^{t_{2 n}}\right)=0$ if $x \cap\{i-1, i\}=\emptyset$ and $t_{i} \neq 0$.

(R-2) $\left(x, y, U_{1}^{t_{1}} \cdots U_{2 n}^{t_{2 n}}\right)=0$ if the local states $x, y$ are far apart from one another, i.e. there is some $i$ such that $\left|x_{i}-y_{i}\right|>1$.

The relations in $(\mathrm{R}-1)$ can be written in terms of indenpotent elements as

$$
I_{x} \cdot U_{i}=0
$$

for any local state $x$ such that $x \cap\{i-1, i\}=\emptyset$. Similarly, the relations in (R-2) can be interpreted as follows. Fix $i \in\{1, \ldots, 2 n-1\}$. Given a local state $x$ such that $i-1 \in x$ but $i \notin x$ we form a new local state $x^{\prime}=(x \backslash\{j-1\}) \cup\{j\}$. Define

$$
R_{i}:=\sum_{\{x \mid i-1 \in x, i \notin x\}}\left(x, x^{\prime}, 1\right) \quad \text { and } \quad L_{i}:=\sum_{\{x \mid i-1 \in x, i \notin x\}}\left(x^{\prime}, x, 1\right) .
$$

Then to say that the relations in (R-2) are satisfied is the same as requiring that $R_{i} \cdot R_{i+1}=0$ and $L_{i+1} \cdot L_{i}=0$ for $i=1, \ldots, 2 n-1$.

5.2. Action of idempotents on partial Kauffman states. Let $K_{[t,+\infty)}$ be an upper diagram with $2 n=|K \cap\{z=t\}|$ endpoints. Given an idempotent element $I_{x} \in I(n)$ and an upper Kauffman state $(\kappa, y)$, set $I_{x} \cdot(\kappa, y)=0$ if $x \neq y$, and $I_{x} \cdot(\kappa, x)=(\kappa, x)$ otherwise. This defines an action of the ring $I(n) \subset \mathcal{C}(n)$ on the vector space $D F K\left(K_{[t,+\infty)}\right)$ generated by upper Kauffman states.

Similarly, given a partial diagram $K_{\left[t_{1}, t_{2}\right]}$ we can consider the algebra $\mathcal{C}\left(n_{2}\right)$ associated to the upper boundary of $K_{\left[t_{1}, t_{2}\right]}$ (the slice at $z=t_{2}$, in particular $2 n_{2}=$ $\left.\left|K \cap\left\{z=t_{2}\right\}\right|\right)$ and the algebra $\mathcal{C}\left(n_{1}\right)$ associated to its lower boundary component $\left(2 n_{1}=\left|K \cap\left\{z=t_{1}\right\}\right|\right)$. Given a partial Kauffman state $(\kappa, x, y)$ of $K_{\left[t_{1}, t_{2}\right]}$ and idempotent states $I_{z^{\prime}} \in I\left(n_{1}\right)$ and $I_{z} \in I\left(n_{2}\right)$ we define

$$
(\kappa, x, y) \cdot I_{z}=\left\{\begin{array}{ll}
(\kappa, x, y) & z=y \\
0 & z \neq y
\end{array} \quad \text { and } \quad I_{z^{\prime}} \cdot(\kappa, x, y)=\left\{\begin{array}{ll}
(\kappa, x, y) & z^{\prime}=x \\
0 & z^{\prime} \neq x
\end{array} .\right.\right.
$$

This turns the vector space generated by partial Kauffman states into a bimodule over $I\left(n_{1}\right)$ and $I\left(n_{2}\right)$. Notice that there are the following identifications of $\mathbb{Z}_{2}$-vector spaces.

Proposition 1. DFK $\left(K_{\left[t_{1},+\infty\right)}\right)=\operatorname{DAFK}\left(K_{\left[t_{1}, t_{2}\right]}\right) \otimes_{I\left(n_{2}\right)} \operatorname{DFK}\left(K_{\left[t_{2},+\infty\right)}\right)$.

Proof. Every upper Kauffman state of $K_{\left[t_{1},+\infty\right)}$ is obtained by gluing an upper Kauffman state $(\kappa, y)$ of $K_{\left[t_{2},+\infty\right)}$ to a partial Kauffman state $\left(\kappa^{\prime}, y, x\right)$ of $K_{\left[t_{1}, t_{2}\right]}$. We 
make a generator $\left(\kappa^{\prime}, x, y\right) \otimes(\kappa, y)$ correspond to the gluing $\left(\kappa^{\prime} * \kappa, x\right)$. For a generator of the form $\left(\kappa^{\prime}, x, y\right) \otimes(\kappa, z)$ with $z \neq y$ one computes $\left(\kappa^{\prime}, x, y\right) \otimes(\kappa, z)=$ $\left(\kappa^{\prime}, x, y\right) \cdot I_{y} \otimes I_{z} \cdot(\kappa, z)=\left(I_{y} \cdot I_{z}\right) \cdot\left(\kappa^{\prime}, x, y\right) \otimes(\kappa, z)=0$ (where we have used the identity: $I_{y} \cdot I_{z}=0$ for $\left.z \neq y\right)$, so we have nothing to map.

Corollary 1. $\widehat{C F K}(K)=A F K\left(K_{(-\infty, t]}\right) \otimes_{I(n)} D F K\left(K_{[t,+\infty)}\right)$.

5.3. Curvature and matchings. $D F K\left(K_{[t,+\infty)}\right)$ and $A F K\left(K_{(-\infty, t]}\right)$ will be respectively regarded as a curved type $D$-structure and $\mathcal{A}_{\infty}$-module over $\mathcal{C}(n)$, where $2 n$ denotes the number of points in the slice $z=t$. The curvature has the following combinatorial interpretation.

For the slice of a knot at $z=t$, we have an associated matching of the $2 n$ intersection points to one another given by how they are connected by the strands lying above the plane $z=t$. This is denoted $M$, and consists of $n$ disjoint pairs $\{i, j\}$ for $i, j \in\{1, \cdots, 2 n\}$. The curvature is given by:

$$
\omega_{M}=\sum_{\{i, j\} \in M} U_{i} U_{j}
$$

Exercise 4. Prove that $\omega_{M}$ defined from a matching $M$ of $\{1, \ldots, 2 n\}$ as above is a central element of $\mathcal{C}(n)$. That is, prove that $\omega_{M} \cdot x=x \cdot \omega_{M}$ for all $x \in \mathcal{C}(n)$.

\section{Structure maps and the Gluing Theorem}

6.1. The type $D$-structure of an upper diagram. We now sketch the definition of the structure map

$$
\delta: D F K\left(K_{[t,+\infty)}\right) \rightarrow \mathcal{C}(n) \otimes_{I(n)} \operatorname{DFK}\left(K_{[t,+\infty)}\right),
$$

of the type $D$-structure associated to an upper diagram $K_{[t,+\infty)}$. Here the ring of indepotents $I(n)$ plays the role of the base ring, while $\mathcal{C}(n)$ the one of the ground $\mathcal{A}_{\infty}$-algebra. The structure constants of $\delta$ will be defined by means of pseudoholomorphic disk counts. First let us interpret upper Kauffman states in terms of intersection points of the appropriate Lagrangian Floer setting.

Let $K \subset \mathbb{R}^{3}$ be a knot. Put $K$ in standard position as in Section 4 and form the doubly pointed Heegaard diagram $\mathcal{H}$ associated to the projection of $K$ on the $x z$-plane as in Section 2.1. Looking at the portion of $\mathcal{H}$ lying above the plane $z=t$ for some $t \in(-10,10)$, we get a genus $g$ surface $\Sigma$ with $2 n$ boundary components $Z_{1}, \ldots, Z_{2 n}$ together with the following data:

(1) a collection $\boldsymbol{\alpha}^{\mathrm{arc}}=\left\{\alpha_{1}^{\mathrm{arc}}, \ldots, \alpha_{2 n-1}^{\text {arc }}\right\}$ of disjoint, properly embedded, oriented arcs, with the $\operatorname{arc} \alpha_{i}^{\text {arc }}$ connecting $Z_{i}$ to $Z_{i+1}$,

(2) a collection of pairwise disjoint, homologically linearly independent, simple closed curves $\boldsymbol{\alpha}=\left\{\alpha_{1}, \ldots, \alpha_{g}\right\}$,

(3) a collection of pairwise disjoint, simple closed curves $\boldsymbol{\beta}=\left\{\beta_{1}, \ldots, \beta_{g+n-1}\right\}$ with the following property: the surface obtained by cutting $\Sigma$ along $\boldsymbol{\beta}$ has $n$ connected components, and each of them contains exactly two of the boundary circles $Z_{1}, \ldots, Z_{2 n}$.

We call the collection of such data an upper Heegaard diagram. Notice that the boundary components of an upper Heegaard diagram $\mathcal{H}^{\text {up }}=\left(\Sigma, \boldsymbol{\alpha}, \boldsymbol{\alpha}^{\text {arc }}, \boldsymbol{\beta}\right)$ come matched together: a component $Z_{i}$ is paired with a component $Z_{j}$ if they can be connected by an arc in the complement of the $\beta$-curves. In the case of knot diagrams 


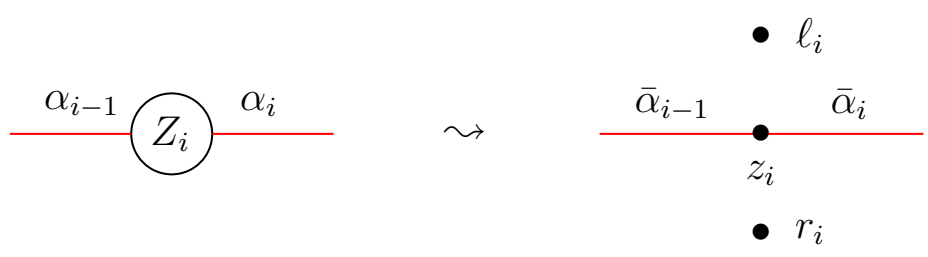

Figure 7 . (Left) Some boundary component $Z_{i}$ of $\Sigma$. (Right) Boundary component $Z_{i}$ filled in and replaced by the point $z_{i}$. Then the points $\ell_{i}$, and $r_{i}$ are placed on opposite sides of $\bar{\alpha}_{i} \cup \bar{\alpha}_{i-1}$. .

this matching is exactly the matching $M$ of $\{1, \ldots, 2 n\}$ in which $\{i, j\} \in M$ iff $i$ and $j$ are the endpoints of a strand of $K_{[t,+\infty)}$. See Figure 8 .

We will be working with pseudo-holomorphic disks in $\operatorname{Sym}^{g+n-1}(\bar{\Sigma})$, where $\bar{\Sigma}$ denotes the closed Riemann surface obtained from $\Sigma$ by pinching its boundary circles. We denote by $z_{1}, \ldots, z_{2 n}$ the punctures of $\bar{\Sigma}$ corresponding to the boundary circles of $\Sigma$. Notice that an alpha arc $\alpha_{i}^{\text {arc }}$ extends to a curve $\bar{\alpha}_{i}^{\text {arc }} \subset \bar{\Sigma}$ with $\partial \bar{\alpha}_{i}^{\text {arc }}=z_{i}-z_{i+1}$. As in the closed case, the $\alpha$ - and the $\beta$-curves specify two Lagrangian submanifolds inside $\operatorname{Sym}^{g+n-1}(\bar{\Sigma})$. More specifically we set $L_{\beta}=\beta_{1} \times \cdots \times \beta_{g+n-1}$ and

$$
L_{\alpha}=\alpha_{1} \times \cdots \times \alpha_{g} \times \operatorname{Sym}^{n-1}\left(\bar{\alpha}_{1}^{\operatorname{arc}} \cup \cdots \cup \bar{\alpha}_{2 n-1}^{\operatorname{arc}}\right) .
$$

Notice that $L_{\alpha}$ is a singular space with singularities at points having more than one coordinate on the same $\alpha$-arc. If we set $L_{\alpha}^{*}=L_{\alpha} \backslash L_{\alpha}^{\text {sing }}$ then we have that

$$
L_{\alpha}^{*}=\bigcup_{\left\{i_{1}, \ldots, i_{n-1}\right\} \subset\{1, \ldots, 2 n-1\}} \alpha_{1} \times \cdots \times \alpha_{g} \times \alpha_{i_{1}}^{\mathrm{arc}} \times \cdots \times \alpha_{i_{n-1}}^{\mathrm{arc}} .
$$

Exercise 5. Prove that the intersection points of $L_{\alpha}^{*} \cap L_{\beta}$ are in one to one correspondence with upper Kauffman states. Show that the local state of the upper Kauffman state associated to an intersection point $\mathbf{x} \in\left(\alpha_{1} \times \cdots \times \alpha_{g} \times \alpha_{i_{1}}^{\mathrm{arc}} \times \cdots \times \alpha_{i_{n-1}}^{\mathrm{arc}}\right) \cap L_{\beta}$ is given by $s(\mathbf{x})=\{1, \ldots, 2 n-1\} \backslash\left\{i_{1}, \ldots, i_{n-1}\right\}$

Let $\mathbf{x}$ and $\mathbf{y} \in L_{\alpha}^{*} \cap L_{\beta}$ be two intersection points. We denote by $\pi_{2}(\mathbf{x}, \mathbf{y})$ the set of homotopy classes of Whitney disks connecting $\mathbf{x}$ to $\mathbf{y}$ with left side lying on $L_{\alpha}$ and right side on $L_{\beta}$. We associate to a homotopy class $\phi \in \pi_{2}(\mathbf{x}, \mathbf{y})$ an algebra element $c(\phi) \in \mathcal{C}(n)$ as follows. In proximity of the punctures $z_{1}, \ldots, z_{2 n}$ place base points $r_{1}, \ldots, r_{2 n}$ and $\ell_{1}, \ldots, \ell_{2 n}$ as suggested by Figure 7 . As in the closed case a homotopy class $\phi \in \pi_{2}(\mathbf{x}, \mathbf{y})$ has multiplicities $r_{i}(\phi)=\#\left|\phi\left(D^{2}\right) \cap V_{r_{i}}\right|$ and $\ell_{i}(\phi)=\#\left|\phi\left(D^{2}\right) \cap V_{\ell_{i}}\right|$. Suppose now $\mathbf{x}$ and $\mathbf{y}$ are two intersection points corresponding to Kauffman states $\left(x, \kappa_{x}\right)$ and $\left(y, \kappa_{y}\right)$ respectively. Define $c(\phi)=\left(x, y, U^{t_{1}} \ldots U^{t_{2 n}}\right) \in C(n)$, where the exponents $t_{1}, \ldots, t_{2 n}$ are chosen so that $c(\phi)$ has weight vector

$$
n(\phi)=\left(\frac{r_{1}(\phi)+\ell_{1}(\phi)}{2}, \ldots, \frac{r_{i}(\phi)+\ell_{i}(\phi)}{2}, \ldots, \frac{r_{2 n}(\phi)+\ell_{2 n}(\phi)}{2}\right) \in \mathbb{Z}^{2 n} .
$$

Lemma 2. Given $\phi_{1} \in \pi_{2}(\mathbf{x}, \mathbf{y})$ and $\phi_{2} \in \pi_{2}(\mathbf{y}, \mathbf{z})$ we have $c\left(\phi_{1}\right) \cdot c\left(\phi_{2}\right)=c\left(\phi_{1} * \phi_{2}\right)$.

Proof. This is true provided that $c\left(\phi_{1}\right) \cdot c\left(\phi_{2}\right)$ and $c\left(\phi_{1} * \phi_{2}\right)$ have the same weight vector. On the other hand we have that $w\left(c\left(\phi_{1} * \phi_{2}\right)\right)=n\left(\phi_{1} * \phi_{2}\right)=n\left(\phi_{1}\right)+n\left(\phi_{2}\right)=$ $w\left(c\left(\phi_{1}\right)\right)+w\left(c\left(\phi_{2}\right)\right)=w\left(c\left(\phi_{1}\right) \cdot c\left(\phi_{2}\right)\right)$. 
We define the structure map $\delta: D F K\left(K_{[t,+\infty)}\right) \rightarrow \mathcal{C}(n) \otimes_{I(n)} D F K\left(K_{[t,+\infty)}\right)$ as follows. Given an intersection point $\mathbf{x} \in L_{\alpha} \cap L_{\beta}$ set

$$
\delta(\mathbf{x})=\sum_{\mathbf{y}} \xi_{\mathbf{x}, \mathbf{y}} \otimes \mathbf{y}
$$

where

$$
\xi_{\mathbf{x}, \mathbf{y}}=\sum_{\phi \in \pi_{2}(\mathbf{x}, \mathbf{y}), \mu(\phi)=1} \#\left(\frac{\mathcal{M}(\phi)}{\mathbb{R}}\right) \cdot c(\phi) .
$$

Theorem 3. $\delta$ is the structure map of an $\omega_{M}$-curved D-structure.

Sketch of the proof. We have to prove that the following relations between the structure constants hold:

$$
\sum_{\mathbf{y}} \xi_{\mathbf{x}, \mathbf{y}} \cdot \xi_{\mathbf{y}, \mathbf{z}}= \begin{cases}\omega_{M} & \text { if } \mathbf{x}=\mathbf{z} \\ 0 & \text { otherwise }\end{cases}
$$

Because of Lemma 2 we have

$$
\sum_{\mathbf{y}} \xi_{\mathbf{x}, \mathbf{y}} \cdot \xi_{\mathbf{y}, \mathbf{z}}=\sum_{\mu(\phi)=2} \lambda(\phi) \cdot c(\phi)
$$

where $\lambda(\phi) \in \mathbb{Z}_{2}$ is given by

$$
\lambda(\phi)=\sum_{\phi_{1} * \phi_{2}=\phi} \#\left(\frac{\mathcal{M}\left(\phi_{1}\right)}{\mathbb{R}}\right) \cdot \#\left(\frac{\mathcal{M}\left(\phi_{2}\right)}{\mathbb{R}}\right) .
$$

This leads us to consider the ends of the moduli space of holomorphic strips in homotopy classes $\phi \in \pi_{2}(\mathbf{x}, \mathbf{z})$ with $\mu(\phi)=2$. For the limit $u$ of a sequence of pseudo-holomorphic disks approaching the boundary of the moduli space $\mathcal{M}(\phi) / \mathbb{R}$ there are a few possibilities:

(1) $u=u_{1} * u_{2}$ is a broken flow-line with $u_{1}$ connecting $\mathbf{x}$ to an intersection point $\mathbf{y} \in L_{\alpha}^{*} \cap L_{\beta}$ and $u_{2}$ connecting $\mathbf{y}$ to $\mathbf{z}$ (with both $u_{1}$ and $u_{2}$ having Maslov index one)

(2) $u=u_{1} * u_{2}$ is a broken flow-line with $u_{1}$ connecting $\mathbf{x}$ to an intersection point $\mathbf{y} \in\left(L_{\alpha} \cap L_{\beta}\right) \backslash\left(L_{\alpha}^{*} \cap L_{\beta}\right)$ and $u_{2}$ connecting $\mathbf{y}$ to $\mathbf{z}$

(3) $\mathbf{x}=\mathbf{z}$ and the boundary of $u$ is entirely mapped on $\overline{L_{\alpha}^{*}}$

(4) $\mathbf{x}=\mathbf{z}$ and the boundary of $u$ is entirely mapped on $L_{\beta}$.

As a consequence of the relations (R-1) and (R-2) we imposed over $\mathcal{C}(n)$ one can see that if degenerations such as (1) and (3) occur, then $c(\phi)=0$. Thus, one only has to consider moduli spaces of strips with $\mu(\phi)=2$ in which the other types of degenerations occur. For these moduli spaces when $\mathbf{x} \neq \mathbf{z}$ we have

$$
0=\#\left|\partial\left(\frac{\mathcal{M}(\phi)}{\mathbb{R}}\right)\right|=\sum_{\phi_{1} * \phi_{2}=\phi} \#\left(\frac{\mathcal{M}\left(\phi_{1}\right)}{\mathbb{R}}\right) \cdot \#\left(\frac{\mathcal{M}\left(\phi_{2}\right)}{\mathbb{R}}\right)=\lambda(\phi) \bmod 2,
$$

proving that $\sum_{\mathbf{y}} \xi_{\mathbf{x}, \mathbf{y}} \cdot \xi_{\mathbf{y}, \mathbf{z}}=0$ when $\mathbf{x} \neq \mathbf{z}$.

On the other hand, when $\mathbf{x}=\mathbf{z}$ there are exactly $n$ homotopy classes of disks $\psi$ with boundary completely lying on $L_{\beta}$. These correspond to the matchings $\{i, j\} \in$ 


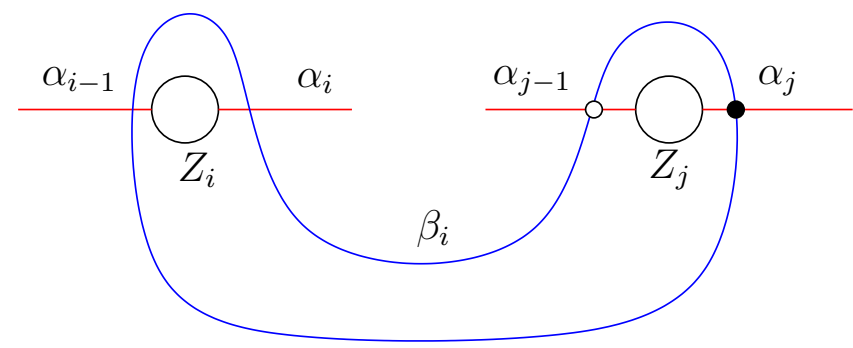

FiguRE 8. The matching $\{i, j\} \in M$ as represented on the upper Heegaard diagram.

$M$, and are represented on the upper Heegaard diagram by the domains of Figure 8. For these domains one computes $c(\phi)=U_{i} \cdot U_{j}$, proving that

$$
\sum_{\mathbf{y}} \xi_{\mathbf{x}, \mathbf{y}} \cdot \xi_{\mathbf{y}, \mathbf{x}}=\sum_{\{i, j\} \in M} U_{i} \cdot U_{j}=\omega_{M}
$$

and we are done.

Remark 4. For sufficiently small $\epsilon>0, C F K\left(K_{[-10+\epsilon,+\infty)}\right)$ is a type $D$-structure over $\mathcal{C}(2)=\mathbb{Z}_{2}[U, V] / U V$. In this case $\omega_{M}=U V=0$, i.e. $C F K\left(K_{[-10+\epsilon,+\infty)}\right)$ is flat. Obviously $C F K\left(K_{[-10+\epsilon,+\infty)}\right)=C F K(K)$ as chain complexes.

6.2. The Gluing Theorem. Similarly but in a somehow more complicated way, one may define the structure maps of an $\mathcal{A}_{\infty}$-module

$$
m_{j}: A F K\left(K_{(-\infty, t]}\right) \otimes \mathcal{C}(n)^{\otimes j-1} \rightarrow A F K\left(K_{(-\infty, t]}\right)
$$

and of a $D A$-bimodule

$$
\delta_{1+j}: D A F K\left(K_{[t, s]}\right) \otimes C(n)^{\otimes j} \rightarrow C(m) \otimes D A F K\left(K_{[t, s]}\right)
$$

where $n$ denotes the number of endpoints at the $z=t$ level and $m$ the number of endpoints at $z=t^{\prime}$. We will not explicitly define these maps here. The main point of this construction is that one can compute the differential of

$$
C F K(K)=\operatorname{AFK}\left(K_{(-\infty, t]}\right) \otimes_{I(n)} \operatorname{DFK}\left(K_{[t,+\infty)}\right),
$$

and more generally the structure map of

$$
\operatorname{DFK}\left(K_{\left[t_{1},+\infty\right)}\right)=\operatorname{DAFK}\left(K_{\left[t_{1}, t_{2}\right]}\right) \otimes_{I\left(n_{1}\right)} \operatorname{DFK}\left(K_{\left[t_{1},+\infty\right)}\right)
$$

by means of Equation (6). More precisely we have the following theorem, whose proof relies on some gluing results about $J$-holomorphic curves.

Theorem 4 (Gluing Theorem, Ozsváth-Szabó). For $-10<t_{1}<t_{2}<+10$ we have an identification of (curved) type D-structures

$$
D F K\left(K_{\left[t_{1},+\infty\right)}\right)=\operatorname{DAFK}\left(K_{\left[t_{1}, t_{2}\right]}\right) \otimes D F K\left(K_{\left[t_{2},+\infty\right)}\right) .
$$

In particular for $t \in(-10,+10)$ one has

$$
C F K(K)=A F K\left(K_{(-\infty, t]}\right) \otimes D F K\left(K_{[t,+\infty)}\right) .
$$

The details of these analytic constructions, as well as a proof of the Gluing Theorem will appear in a forthcoming work of Ozsváth and Szabó. 


\section{ThE BIMODUles OF SOME ELEMENTARY CONFIGURATIONS}

Any knot $K \subset \mathbb{R}^{3}$ can be presented by a diagram obtained by concatenating elementary configurations as in Figures 9 to 11 and 13. This can be done by suitably modifying a knot projection in standard position as in Figure 12 below. We now explicitly describe the structure maps of the curved $D A$-bimodules associated to these partial diagrams. Based on the Gluing Theorem one can then use the maps coming from these elementary computations to express the differential of knot Floer homology. This leads to a combinatorial formulation of knot Floer homology (see Section section 7.5 below) alternative to grid homology [5].

7.1. Maxima. While scanning a knot projection in standard position all maxima appear at the same level, say $z=+10$. The portion of the diagram in between the slice $z=10$ and $z=10-\epsilon$ will then look like Figure 9. In this case there is only one upper Kauffman state, corresponding to the local state $x$ taking the intervals: $(1,2),(3,4), \ldots,(2 n-1,2 n)$. See Figure 9. Idempotents act on $x$ in the obvious way: $I_{y} \cdot x=x$ if $x=y$, zero otherwise. Furthermore, one computes $\delta(x)=0$. As such, the module is isomorphic to $\mathbb{Z}_{2}$.

7.2. No critical points, no gain. To make a sanity check, let us consider the case of the trivial diagram in which $2 n$ strands run parallel to each other as in Figure 10. In this case $D A F K$ is identified with the ring of the idempotents $I(n) \subset \mathcal{C}(n)$ with the left-multiplication action. The structure maps $\delta_{1+j}: I(n) \otimes \mathcal{C}(n)^{\otimes j} \rightarrow$ $\mathcal{C}(n)$ are all zero except for $\delta_{2}: I(n) \otimes \mathcal{C}(n) \rightarrow \mathcal{C}(n)$. For the latter we have $\delta_{2}\left(I_{x} \otimes\left(x, y, U_{1}^{t_{1}} \ldots U_{2 n}^{t_{2 n}}\right)\right)=\left(x, y, U_{1}^{t_{1}} \ldots U_{2 n}^{t_{2 n}}\right) \otimes I_{y}$, and zero otherwise.

Exercise 6. Let $\left(X, \delta_{X}\right)$ be the type $D$-structure associated to an upper knot diagram with $2 n$ endpoints. Prove that $\left(I(n), \delta_{1+*}\right) \otimes\left(X, \delta_{X}\right)=\left(X, \delta_{X}\right)$. (Hint:

$$
\delta_{I(n) \otimes X}=\sum_{j=0}^{+\infty}\left(\delta_{1+j} \otimes i d_{X}\right) \circ\left(i d_{I(n)} \otimes \delta_{X}^{j}\right)=\left(\delta_{2} \otimes i d_{X}\right) \circ\left(i d_{I(n)} \otimes \delta_{X}\right),
$$

so one has just to prove that $\left(\delta_{2} \otimes i d_{X}\right) \circ\left(i d_{I(n)} \otimes \delta_{X}\right)(1 \otimes \mathbf{x})=1 \otimes \delta_{X}(\mathbf{x})$ for any generator $\mathbf{x}=(\kappa, x)$ of $X$. Recall that in $I(n)$ we have $1=\sum_{\text {local states }} I_{x}$.)

7.3. Local Minima. Suppose now that while scanning a knot $K$ we encounter a local minimum configuration between two "consecutive" levels $z=t_{1}$ and $z=t_{2}$. See Figure 11. The generators of $\mathcal{M}_{n+1}=D A F K\left(K_{\left[t_{1}, t_{2}\right]}\right)$ of such a configuration look as in Figure 11. We now describe the $\delta_{1+j}$ maps.

Denote by $M$ the matching of the upper portion of the diagram $K_{\left[t_{2},+\infty\right)}$. Since $K$ is a knot the first two endpoints of $K_{\left[t_{2},+\infty\right)}$ are not matched together. Let $s$ and $t \in\{2, \ldots, 2 n+2\}$ be the endpoints matched with 1 and 2 according to $M$. Notice that while passing to the level $z=t_{1}$ we get a new matching $M^{\prime}$ in which $s$ and $t$ are matched together, and $\{i, j\} \in M^{\prime}$ iff $\{i, j\} \in M \backslash\{\{1, s\},\{2, t\}\}$.

Let $\mathbf{x}=\left(\emptyset, \mathbf{x}^{\text {in }}, \mathbf{x}^{\text {out }}\right)$ and $\mathbf{y}=\left(\emptyset, \mathbf{y}^{\text {in }}, \mathbf{y}^{\text {out }}\right)$ be generators of $D A F K$, and $a_{1}=$ $\left(a_{1}^{\text {in }}, a_{1}^{\text {out }}, m_{1}\left(U_{1}, \ldots, U_{2 n+2}\right)\right), \ldots, a_{j}=\left(a_{j}^{\text {in }}, a_{j}^{\text {out }}, m_{j}\left(U_{1}, \ldots, U_{2 n+2}\right)\right)$ denote algebra elements in $\mathcal{C}(n+1)$. Write

$$
\delta_{j+1}\left(\mathbf{x} \otimes a_{1} \otimes \cdots \otimes a_{j}\right)=\sum_{\mathbf{y}} \xi_{\mathbf{x}, \mathbf{y}}\left(a_{1}, \ldots, a_{j}\right) \cdot \mathbf{y}
$$




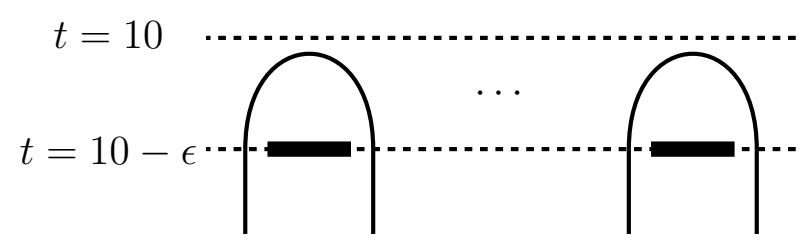

FiguRE 9. All of the maxima are on the same level between $z=$ 10 and $z=10-\epsilon$. Any knot in standard position can be suitably modified to look like this. Note that there is only one upper Kauffman state, which is the one pictured here where we use a dot to denote an occupied region.

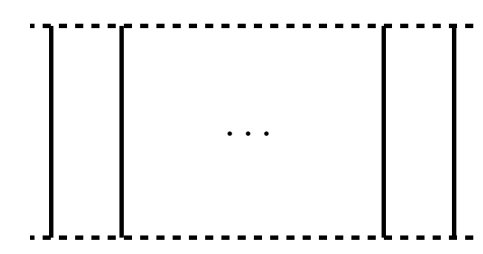

Figure 10. The trivial diagram with $2 n$ strands running parallel.

with $\xi_{\mathbf{x}, \mathbf{y}}\left(a_{1}, \ldots, a_{j}\right) \in \mathcal{C}(n)$. The coefficients $\xi_{\mathbf{x}, \mathbf{y}}\left(a_{1}, \ldots, a_{j}\right)$ are usually zero unless $j=2 m+1$ is odd,

$$
\mathbf{x}^{i n}=a_{1}^{i n}, \quad a_{1}^{\text {out }}=a_{2}^{\text {in }}, \quad \ldots, \quad a_{2 m}^{\text {out }}=a_{2 m+1}^{\text {in }}, \quad a_{2 m+1}^{\text {out }}=\mathbf{y}^{\text {in }},
$$

and the weight vectors of the $a_{i}$ 's are of the form

$$
\begin{array}{ll}
w\left(a_{2 \ell-1}\right)=\left(0, \frac{1}{2}+r_{i}, \ldots\right) & \ell=1, \ldots, m \\
w\left(a_{2 \ell}\right)=\left(1+p_{\ell}, 0, \ldots\right) & \ell=1, \ldots, m .
\end{array}
$$

In this case we take $\xi_{\mathbf{x}, \mathbf{y}}\left(a_{1}, \ldots, a_{2 m+1}\right)=\left(\mathbf{x}^{\text {out }}, \mathbf{y}^{\text {out }}, U_{1}^{t_{1}} \ldots U_{2 n}^{t_{2} n}\right)$, where the exponents $t_{1}, \ldots, t_{2 m} \in \mathbb{Z}_{\geq 0}$ are chosen so that $\xi_{\mathbf{x}, \mathbf{y}}\left(a_{1}, \ldots, a_{2 m+1}\right)$ has weight vector $w=\left(w_{1}, \ldots, w_{2 n}\right)$ with components:

$$
\begin{array}{ll}
w_{i}=\sum_{k} w_{i+2}\left(a_{k}\right) & \text { if } i \neq t-2, s-2 \\
w_{t-2}=\sum_{k} w_{i+2}\left(a_{k}\right)+\sum_{\ell} p_{\ell} & \\
w_{s-2}=\sum_{k} w_{i+2}\left(a_{k}\right)+\sum_{\ell} r_{\ell} . &
\end{array}
$$

In particular the map $\delta_{1+j}: \mathcal{M}_{n+1} \rightarrow \mathcal{C}(n+1)^{\otimes j} \rightarrow \mathcal{C}(n)$ vanishes for $j$ even.

7.4. Crossings. We now define the $D A$-bimodules associated to the crossings. We will deal with the case of positive crossings first, and the case of a negative crossing will just be a slight variation of the same formulation.

7.4.1. Positive crossings. Positive crossings are distinguished from negative crossings as in Figure 13. We construct a $D A$-bimodule $D A F K=P$ over $\mathcal{C}(n)$. This has four types of generators, which we write as $N, S, W$, and $E$ corresponding to 


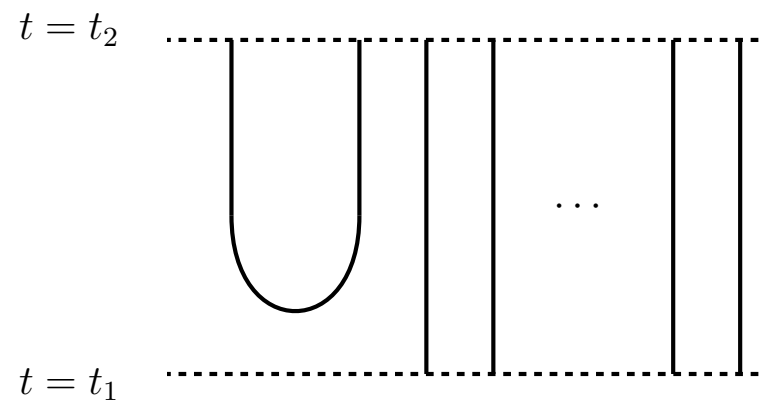

Figure 11. A local minimum between two levels $z=t_{1}$ and $z=t_{2}$. Any local minimum can be made to look like this one as in Figure 12.
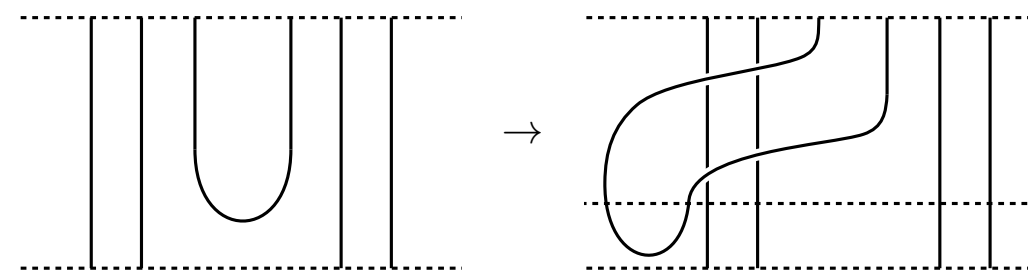

FIgURE 12. For any local minimum in the slice $K_{\left[t_{1}, t_{2}\right]}$, we can manipulate the knot such that it instead has a local minimum of the form in Figure 11. This of course comes at the cost of additional crossings.
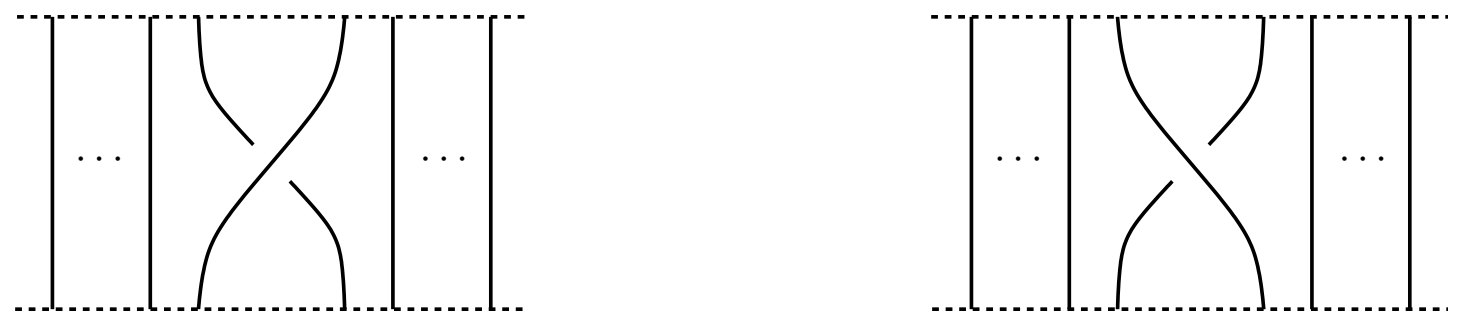

FiguRE 13. (Left) The portion of the knot $K_{\left[t_{1}, t_{2}\right]}$ contains a positive crossing. (Right) The portion of the knot $K_{\left[t_{1}, t_{2}\right]}$ contains a negative crossing.

the possibilities near the crossing as depicted in Figure 14. These are distinguished from one another by their partial Kauffman states. We now specify $\delta_{1}, \delta_{2}$, and $\delta_{3}$, the higher $\delta_{i}$ 's vanish.

We first explicitly write:

$$
\delta_{1}(N)=0, \quad \delta_{1}(E)=R_{2} \otimes S, \quad \delta_{1}(S)=0 \text { and } \delta_{1}(W)=L_{1} \otimes S
$$




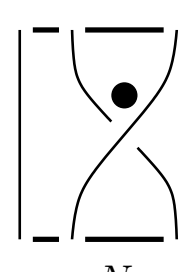

$N$

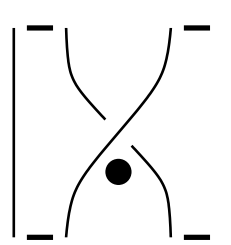

$S$

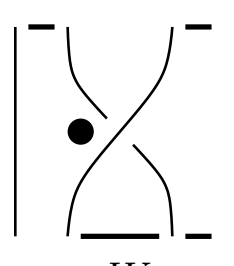

$W$

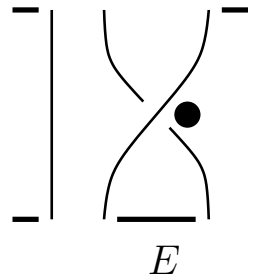

FiguRE 14. The partial Kauffman states associated to the four types of positive crossings which generate the $D A$-bimodule.

The structure map $\delta_{2}$ is specified according to the following:

$$
\begin{aligned}
& \delta_{2}(S)=1 \otimes 1 \otimes S \\
& \delta_{2}(E)=1 \otimes 1 \otimes E+1 \otimes L_{2} \otimes N+R_{2} R_{1} \otimes R_{2} U_{2} \otimes N+R_{2} R_{2} \otimes U_{2} \otimes W \\
& \delta_{2}(W)=1 \otimes 1 \otimes E+1 \otimes R_{1} \otimes N+L_{1} L_{2} \otimes L_{2} U_{1} \otimes N+L_{1} L_{2} \otimes U_{1} \otimes E \\
& \delta_{2}(N)=U_{1} \otimes R_{2} \otimes E+L_{1} L_{2} \otimes L_{1} \otimes E+U_{2} \otimes L_{1} \otimes W+R_{2} R_{1} \otimes R_{2} \otimes W \\
& \quad+1 \otimes 1 \otimes N+L_{1} L_{2} \otimes L_{1} L_{2} \otimes N+R_{2} R_{1} \otimes R_{2} R_{1} \otimes N
\end{aligned}
$$

These can be extend using some local extension rules:

- If $b \otimes Y$ appears with non-zero coefficient in $\delta_{2}(N, a)$, then $\left(b U_{2}\right) \otimes Y$ appears with non-zero coefficient in $\delta_{2}\left(N, a U_{1}\right)$ and $\left(b U_{1}\right) \otimes Y$ appears with non-zero coefficient in $\delta_{2}\left(N, a U_{2}\right)$.

- If $b \otimes Y$ appears with non-zero coefficient in $\delta_{2}(W, a)$, then $\left(U_{2} b\right) \otimes Y$ appears with non-zero coefficient in $\delta_{2}\left(W, U_{1} a\right)$.

- If $b \otimes Y$ appears with non-zero coefficient in $\delta_{2}(E, a)$, then $\left(U_{1} b\right) \otimes Y$ appears with non-zero coefficient in $\delta_{2}\left(E, U_{2} a\right)$.

for example, $\delta_{2}\left(W, U_{1}\right)=U_{2} \otimes W+L_{1} L_{2} \otimes E$.

Finally we specify $\delta_{3}$. We will call an algebra element elementary if it is of the form $p \cdot e$ for $p$ a monomial in $U_{1}$ and $U_{2}$, and

$$
e \in\left\{1, L_{1}, R_{1}, L_{2}, R_{2}, L_{1} L_{2}, R_{2} R_{1}\right\}
$$

So let $a_{1}$ and $a_{2}$ be elementary algebra elements such that $a_{1} \otimes a_{2} \neq 0$. Also suppose that $U_{1} U_{2}$ does not divide either $a_{1}$ nor $a_{2}$. Then $\delta_{3}\left(S, a_{1}, a_{2}\right)$ is the sum of the following terms:

- $R_{1} U_{1}^{t} \otimes E$ if $\left(a_{1}, a_{2}\right)=\left(R_{1}, R_{2} U_{2}^{t}\right)$ and $t \geq 0$.

- $L_{2} U_{1}^{t} U_{2}^{n} \otimes E$ if $\left(a_{1}, a_{2}\right) \in$

$$
\begin{array}{ll}
\left\{\left(U_{1}^{n+1}, U_{2}^{t}\right),\left(R_{1} U_{1}^{n}, L_{1} U_{2}^{t}\right),\left(L_{2} U_{1}^{n+1}, R_{2} U_{2}^{t-1}\right)\right\} & \text { when } 0 \leq n<t \\
\left\{\left(U_{2}^{t}, U_{1}^{n+1}\right),\left(R_{1} U_{2}^{t}, L_{1} U_{1}^{n}\right),\left(L_{2} U_{2}^{t-1}, R_{2} U_{1}^{n+1}\right)\right\} & \text { when } 1 \leq t \leq n
\end{array}
$$

- $L_{2} U_{2}^{n} \otimes W$ if $\left(a_{1}, a_{2}\right)=\left(L_{2}, L_{1} U_{1}^{n}\right)$ and $n \geq 0$.

- $R_{1} U_{1}^{t} U_{2}^{n} \otimes W$ if $\left(a_{1}, a_{2}\right) \in$

$$
\begin{array}{ll}
\left\{\left(U_{1}^{t+1}, U_{1}^{n}\right),\left(L_{2} U_{2}^{t}, R_{2} U_{1}^{n}\right),\left(R_{1} U_{2}^{t+1}, L_{1} U_{1}^{n-1}\right)\right\} & \text { when } 0 \leq t<n \\
\left\{\left(U_{1}^{n}, U_{2}^{t+1}\right),\left(L_{2} U_{1}^{n}, R_{2} U_{2}^{t}\right),\left(R_{1} U_{1}^{n-1}, L_{1} U_{2}^{t+1}\right)\right\} & \text { when } 1 \leq n \leq t
\end{array}
$$


- $L_{2} U_{1}^{t} U_{2}^{n} \otimes N$ if $\left(a_{1}, a_{2}\right) \in$

$$
\begin{array}{rr}
\left\{\left(U_{1}^{n+1}, L_{2} U_{2}^{t}\right),\left(R_{1} U_{1}^{n}, L_{1} L_{2} U_{2}^{t}\right),\left(R_{1} U_{2}^{t+1}, U_{1}^{n}\right)\right\} & \text { when } 0 \leq n<t \\
\left\{\left(L_{2} U_{2}^{t}, U_{1}^{n+1}\right),\left(U_{2}^{t}, L_{2} U_{1}^{n+1}\right)\left(R_{1} U_{2}^{t}, L_{1} L_{2} U_{1}^{n}\right)\right\} & \text { when } 1 \leq t \leq n \\
\left\{\left(L_{2}, U_{1}^{n+1}\right)\right\} & \text { when } 0=t \leq n
\end{array}
$$

- $R_{1} U_{1}^{t} U_{2}^{n} \otimes N$ if $\left(a_{1}, a_{2}\right)$ is in the following list:

$$
\begin{array}{rr}
\left\{\left(U_{2}^{t+1}, R_{1} U_{1}^{n}\right),\left(L_{2} U_{2}^{t}, R_{2} R_{1} U_{1}^{n}\right),\left(R_{1} U_{2}^{t+1}, U_{1}^{n}\right)\right\} & \text { when } 0 \leq t<n \\
\left\{\left(R_{1} U_{1}^{n}, U_{2}^{t+1}\right),\left(U_{1}^{n}, R_{1} U_{2}^{t+1}\right),\left(L_{2} U_{1}^{n}, R_{2} R_{1} U_{2}^{t}\right)\right\} & \text { when } 1 \leq n \leq t \\
\left\{\left(R_{1}, U_{2}^{t+1}\right)\right\} & \text { when } 0=n \leq t
\end{array}
$$

7.4.2. Negative crossings. We now construct a $D A$-bimodule $N$ over $\mathcal{C}(n)$ to be associated to a negative crossing. These are distinguished from positive crossings as in Figure 13. This bimodule has the same four types of generators $N, S, E$, and $W$ and is just a tweaked version of $P$. Define a map $o: \mathcal{C}(n) \rightarrow \mathcal{C}(n)$ to satisfy $o(a \cdot b)=o(b) \cdot o(a)$ and in particular take:

$$
\begin{array}{cr}
o\left(I_{x}\right)=I_{x} & o\left(U_{t}\right)=U_{t} \\
o\left(L_{t}\right)=R_{t} & o\left(R_{t}\right)=L_{t} .
\end{array}
$$

If $\delta_{1}^{1}(X)=b \otimes Y$ in $P$, then $\delta_{1}(Y)=o(b) \otimes X$ in $N$. If $\delta_{2}(X, a)=o(b) \otimes Y$ in $P$, then $\delta_{2}(Y, o(a))=o(b) \otimes X$ in $N$. Finally, if $\delta_{3}\left(X, a_{1}, a_{2}\right)=b \otimes Y$ in $P$, then $\delta_{3}\left(Y, o\left(a_{2}\right), o\left(a_{1}\right)\right)=o(b) \otimes X$.

7.5. A final remark. In principle one can forget about the analytic theory and define knot Floer homology as follows. Start from a knot projection $D$ in standard position as in the beginning of Section 4.1. Up to isotopy we can suppose that the crossings $c_{1}, \ldots, c_{m}$ of $D$ all have different $z$-coordinate. Let $\overline{\mathcal{M}}$ be the type $D$-structure of Section $6.1, \underline{\mathcal{M}}=\mathcal{M}_{n} \otimes \mathcal{M}_{n-1} \otimes \cdots \otimes \mathcal{M}_{2}$ be the $D A$-bimodule obtained by taking the box tensor product of the $D A$-bimodules of Section 7 , and let $\mathcal{X}_{i}$ denote the $D A$-bimodule associated to $K_{\left[z\left(c_{i}\right)-\epsilon, z\left(c_{i}\right)+\epsilon\right]}$ as in Section 7.4 above. Define

$$
C(D)=\overline{\mathcal{M}} \otimes \mathcal{X}_{1} \otimes \cdots \otimes \mathcal{X}_{m} \otimes \underline{\mathcal{M}}
$$

Obviously the definition of $C(D)$ only makes reference to the diagram $D$, no choice of a generic almost-complex structure needs to be done, and no holomorphic disk count is involved. In fact, in [6] Ozsváth and Szabó proved that the chain homotopy type of $C(D)$ does not change if $D$ is changed by any of the Reidemeister moves. This was done locally at level of $D A$-bimodules, by proving that the braid relations are satisfied.

Theorem 5 (Ozsváth-Szabó). The chain homotopy type of $C(D)$ is a knot invariant.

Notice that the chain complexes coming from this construction are more "economic" than the one coming from the combinatorial formulation of [5]. 


\section{REFERENCES}

[1] D. Auroux, A beginner's introduction to Fukaya categories, ArXiv e-prints (January 2013), available at 1301.7056 .

[2] K. Frøyshov, Compactness and gluing theory for monopoles, Geometry \& Topology Monographs, vol. 15, Geometry \& Topology Publications, Coventry, 2008. MR2465077

[3] P. Kronheimer and T. Mrowka, Monopoles and three-manifolds, New Mathematical Monographs, Cambridge University Press, 2007.

[4] R. Lipshitz, P. Ozsváth, and D. Thurston, Bordered Heegaard Floer homology: Invariance and pairing, ArXiv e-prints (October 2008), available at 0810.0687.

[5] P. Ozsváth, A. Stipsicz, and Z. Szabó, Grid homology for knots and links, AMS Mathematical Surveys and Monographs, 2015.

[6] P. Ozsváth and Z. Szabó, Kauffman states, bordered algebras, and a bigraded knot invariant. arXiv:1603.06559.

[7] _ Holomorphic disks and knot invariants, Advances in Mathematics 8 (2004), 58-116.

[8] _ Holomorphic disks and topological invariants for closed three manifolds, Annals of Mathematics 159 (2004), 1027-1158.

[9] _ An introduction to Heegaard Floer homology, Floer homology, gauge theory, and lowdimensional topology, 2006, pp. 3-27. MR2249247

[10] T. Perutz, A remark on Kähler forms on symmetric products of Riemann surfaces, ArXiv Mathematics e-prints (January 2005), available at math/0501547.

[11] J. Rasmussen, Floer homology and knot complements, ArXiv Mathematics e-prints (June 2003), available at math/0306378.

Central European University

E-mail address: alfieri_antonio@phd.ceu.edu

UNIVERSITY OF CALIFORNIA, BERKELEY

E-mail address: jacksontvandyke@berkeley.edu 